\title{
Spectral collocation and a two-level continuation scheme for dipolar Bose-Einstein condensates
}

\author{
B.-W. Jeng ${ }^{a, *}$, C.-S. Chien ${ }^{b, \dagger}$, I.-L. Chern ${ }^{c, \ddagger}$ \\ ${ }^{a}$ Department of Mathematics Education, \\ National Taichung University of Education, Taichung 403, Taiwan \\ ${ }^{b}$ Department of Computer Science and Information Engineering, \\ Chien Hsin University of Science and Technology, Zhongli 320, Taiwan \\ ${ }^{c}$ Department of Mathematics, \\ National Taiwan University, Taipei 106, Taiwan
}

\begin{abstract}
We exploit the high accuracy of spectral collocation methods in the context of a two-level continuation scheme for computing ground state solutions of dipolar Bose-Einstein condensates (BEC), where the first kind Chebyshev polynomials and Fourier sine functions are used as the basis functions for the trial function space. The governing Gross-Pitaevskii equation (or Schrödinger equation) can be reformulated as a Schrödinger-Poisson (SP) type system [13]. The two-level continuation scheme is developed for tracing the first solution curves of the SP system, which in turn provide an appropriate initial guess for the Newton method to compute ground state solutions for 3D dipolar BEC. Extensive numerical experiments on 3D dipolar BEC and dipolar BEC in optical lattices are reported.
\end{abstract}

Keywords: spectral method, Gross-Pitaevskii equation, dipolar BEC, periodic potential.

*Supported by the National Science Council of R.O.C.(Taiwan) through Project NSC 102-2115-M-142002 .

${ }^{\dagger}$ Corresponding author. Supported by the National Science Council of R.O.C.(Taiwan) through Project NSC 101-2115-M-231-001-MY2. E-mail address: cschien@uch.edu.tw, cschien@amath.nchu.edu.tw (C.-S. Chien), bwjeng@mail.ntcu.edu.tw (B.-W. Jeng), chern@math.ntu.edu.tw (I.-L. Chern).

${ }^{\ddagger}$ Supported by the National Science Council of R.O.C.(Taiwan) through Project NSC 99-2811-M-002-189. 


\section{Introduction}

In past years, a successful experimental result has been obtained on dipolar Bose-Einstein condensates (BECs), where a BEC of ${ }^{52} \mathrm{Cr}$ atoms has been realized [1]. The achievement allows experimental investigations of the unique properties of dipolar BEC. The experimental breakthrough on cooling and trapping of molecules [2], on photoassociation [3], and on Feshbach resonances of binary mixtures [4] have opened up new research areas in the atomic physics community [5-11].

The 3D dipolar BEC at zero temperature trapped in a harmonic potential $V(\mathbf{x})=$ $\frac{m}{2}\left(\omega_{x}^{2} x^{2}+\omega_{y}^{2} y^{2}+\omega_{z}^{2} z^{2}\right)$ is well described by the macroscopic wave function $\psi(\mathbf{x}, t)$ whose evolution is governed by the Gross-Pitaevskii equation (GPE) $[5,12]$

$$
\mathbf{i} \hbar \partial_{t} \psi(\mathbf{x}, t)=\left[-\frac{\hbar^{2}}{2 m} \Delta+V(\mathbf{x})+U_{0}|\psi(\mathbf{x}, t)|^{2}+V_{\text {dip }}(\mathbf{x}) *|\psi(\mathbf{x}, t)|^{2}\right] \psi(\mathbf{x}, t), \mathbf{x} \in \mathbb{R}^{3}, t>0
$$

where $\hbar$ is the Planck constant, $t$ is the time variable, $\mathbf{x}=(x, y, z)^{T} \in \mathbb{R}^{3}, m$ is the mass of a dipolar particle, $\omega_{x}, \omega_{y}$, and $\omega_{z}$ are the trap frequencies in the $x_{-}, y$-, and $z$-direction, respectively. The parameter $U_{0}=\frac{4 \pi \hbar^{2} a_{s}}{m}$ denotes local (or short-range) interaction between dipoles in the condensates with $a_{s}$ the $s$-wave scattering length, $V_{\text {dip }}$ denotes the long-range dipolar interaction between two dipoles which is given by

$$
V_{\text {dip }}(\mathbf{x})=\frac{\mu_{0} \mu_{\text {dip }}^{2}}{4 \pi} \frac{1-3(\mathbf{x} \cdot \mathbf{n})^{2} /|\mathbf{x}|^{2}}{|\mathbf{x}|^{3}}=\frac{\mu_{0} \mu_{\text {dip }}^{2}}{4 \pi} \frac{1-3 \cos ^{2} \theta}{|\mathbf{x}|^{3}}, \mathbf{x} \in \mathbb{R}^{3},
$$

where $\mu_{0}$ is the vacuum magnetic permeability, $\mu_{\text {dip }}$ is the permanent magnetic dipole moment (e.g., $\mu_{\text {dip }}=6 \mu_{B}$ for ${ }^{52}$ Cr with $\mu_{B}$ being the Bohr magneton), $\mathbf{n}=\left(n_{1}, n_{2}, n_{3}\right)^{T} \in \mathbb{R}^{3}$ is the dipole axis (or dipole moment) with $\|\mathbf{n}\|_{2}=1$, and $\theta$ is the angle between the dipole axis $\mathbf{n}$ and position vector $\mathbf{x}$. The wave function $\psi$ is normalized according to

$$
\|\psi\|^{2}:=\int_{\mathbb{R}^{3}}|\psi(\mathbf{x}, t)|^{2} d \mathbf{x}=M
$$

where $M$ is the total number of dipolar particles in the dipolar BEC.

By introducing the dimensionless variables [13], $t \rightarrow \frac{t}{\omega_{0}}$ with $\omega_{0}=\min \left\{\omega_{x}, \omega_{y}, \omega_{z}\right\}$, $\mathbf{x} \rightarrow a_{0} \mathbf{x}$ with $a_{0}=\sqrt{\hbar / m \omega_{0}}, \psi \rightarrow \frac{\sqrt{M} \psi}{a_{0}^{3 / 2}}$, Eqs. (1.1) and (1.3) are expressed as

$$
\mathbf{i} \partial_{t} \psi(\mathbf{x}, t)=\left[-\frac{1}{2} \Delta+V(\mathbf{x})+\beta|\psi(\mathbf{x}, t)|^{2}+\lambda\left(U_{\text {dip }}(\mathbf{x}) *|\psi(\mathbf{x}, t)|^{2}\right)\right] \psi(\mathbf{x}, t), \mathbf{x} \in \mathbb{R}^{3}, t>0
$$




$$
\|\psi\|^{2}=\int_{\mathbb{R}^{3}}|\psi(\mathbf{x}, t)|^{2} d \mathbf{x}=1
$$

where $V(\mathbf{x})=\frac{1}{2}\left(\gamma_{x}^{2} x^{2}+\gamma_{y}^{2} y^{2}+\gamma_{z}^{2} z^{2}\right)$ is the dimensionless harmonic trapping potential with $\gamma_{x}=\omega_{x} / \omega_{0}, \gamma_{y}=\omega_{y} / \omega_{0}$, and $\gamma_{z}=\omega_{z} / \omega_{0}, \beta=\frac{M U_{0}}{\hbar \omega_{0} a_{0}^{3}}=\frac{4 \pi a_{s} M}{a_{0}}, \lambda=\frac{m M \mu_{0} \mu_{\text {dip }}^{2}}{3 \hbar^{2} a_{0}}$, and the dimensionless long-range dipolar interaction potential $U_{\text {dip }}$ is given as

$$
U_{\text {dip }}(\mathbf{x})=\frac{3}{4 \pi} \frac{1-3(\mathbf{x} \cdot \mathbf{n})^{2} /|\mathbf{x}|^{2}}{|\mathbf{x}|^{3}}=\frac{3}{4 \pi} \frac{1-3 \cos ^{2} \theta}{|\mathbf{x}|^{3}}, \mathbf{x} \in \mathbb{R}^{3} .
$$

Recently, Bao et al. [13] decoupled the two-body dipolar interaction potential into shortrange (or local) and long-range interactions (or repulsive and attractive interactions), and transformed Eq. (1.4) into a Gross-Pitaevskii-Poisson or Schrödinger-Poisson (SP) type system of the following form

$$
\begin{gathered}
\mathbf{i} \partial_{t} \psi(\mathbf{x}, t)=\left[-\frac{1}{2} \Delta+\right. \\
\left\{\begin{array}{l}
\left.-\Delta(\mathbf{x})+(\beta-\lambda)|\psi(\mathbf{x}, t)|^{2}-3 \lambda \widetilde{\varphi}(\mathbf{x}, t)\right] \psi(\mathbf{x}, t), \mathbf{x} \in \mathbb{R}^{3}, t>0, \\
\widetilde{\varphi}(\mathbf{x}, t)=\left.\partial_{\mathbf{n n}} \varphi(\mathbf{x}, t)\right|^{2}, \quad \lim _{|\mathbf{x}| \rightarrow \infty} \varphi(\mathbf{x}, t)=0,
\end{array}\right.
\end{gathered}
$$

where $\partial_{\mathbf{n}}=\mathbf{n} \cdot \nabla=n_{1} \partial_{x}+n_{2} \partial_{y}+n_{3} \partial_{z}, \partial_{\mathbf{n n}}=\partial_{\mathbf{n}}\left(\partial_{\mathbf{n}}\right)$. The decoupled short-range and long-range interactions of the dipolar interaction potential are attractive and repulsive, respectively, when $\lambda>0$; and are repulsive and attractive, respectively, when $\lambda<0$. Moreover, the total energy per particle is

$$
E(\psi)=E_{\text {kin }}(\psi)+E_{\text {pot }}(\psi)+E_{\text {int }}(\psi)+E_{\text {dip }}(\psi)
$$

where

$$
\begin{aligned}
& E_{\text {kin }}(\psi)=\frac{1}{2} \int_{\mathbb{R}^{3}}|\nabla \psi|^{2} d \mathbf{x}, \quad E_{\text {pot }}(\psi)=\int_{\mathbb{R}^{3}} V(\mathbf{x})|\psi|^{2} d \mathbf{x}, \\
& E_{\text {int }}(\psi)=\frac{\beta}{2} \int_{\mathbb{R}^{3}}|\psi|^{4} d \mathbf{x}, \quad E_{\text {dip }}(\psi)=\frac{\lambda}{2} \int_{\mathbb{R}^{3}}\left(-|\psi|^{4}+3\left|\partial_{\mathbf{n}} \nabla \varphi\right|^{2}\right) d \mathbf{x},
\end{aligned}
$$

are kinetic, potential, interaction, and dipolar energies, respectively. Substituting the formula [14]

$$
\psi(\mathbf{x}, t)=e^{-\mathbf{i} \mu t} \phi(\mathbf{x}), \mathbf{x} \in \mathbb{R}^{3}, t \geq 0
$$


into Eqs. (1.7)-(1.8) and the constraint (1.5), we obtain the nonlinear eigenvalue problem

$$
\begin{aligned}
& {\left[-\frac{1}{2} \Delta+V(\mathbf{x})+(\beta-\lambda)|\phi(\mathbf{x})|^{2}-3 \lambda \widetilde{\varphi}(\mathbf{x})\right] \phi(\mathbf{x})=\mu \phi(\mathbf{x}), \mathbf{x} \in \mathbb{R}^{3},} \\
& \|\phi\|^{2}=\int_{\mathbb{R}^{3}}|\phi(\mathbf{x})|^{2} d \mathbf{x}=1 \\
& -\Delta \varphi(\mathbf{x})=|\phi(\mathbf{x})|^{2}, \quad \lim _{|\mathbf{x}| \rightarrow \infty} \varphi(\mathbf{x})=0 \\
& \widetilde{\varphi}(\mathbf{x})=\partial_{\mathbf{n n}} \varphi(\mathbf{x}) .
\end{aligned}
$$

Eq. (1.11) is a stationary state SP type system. A different type of SP system, called selfconsistent SP system, is described in [14] with totally different physical meaning. In this system we are mainly interested in computing the first few eigenpairs of the associated selfconsistent SP eigenvalue problem. The numerical study for the self-consistent SP eigenvalue problem can be found in [15]. Recently, Chien et al. [16] described a two-grid finite element discretization scheme, which is a time-independent approach for computing wave functions of the self-consistent SP system.

Recently, various numerical methods have been proposed for investigating certain physical phenomena of BEC. See e.g., [17-19]. The study of Eq. (1.11) in 3D, and in 1D and 2D, was reported in [13] and [20,21], respectively. Since nearly all particles of BEC fall into the ground state of the trap, the macroscopic wave function $\phi(\mathbf{x})$ decays to zero rapidly as $|\mathbf{x}| \rightarrow \infty$. So we replace the whole domain $\mathbb{R}^{3}$ by a finite large domain $\Omega=(-L, L)^{3}$ with $L>0$, and impose the zero Dirichlet boundary conditions on the functions $\phi(\mathbf{x})$ and $\varphi(\mathbf{x})$. In this paper, we describe a novel two-level continuation scheme for tracing the first solution curves of Eq. (1.11) and dipolar BEC in optical lattices, which can provide an appropriate initial guess for the Newton method to compute ground state solutions of the SP system. The outer continuation determines the first consecutive bifurcation points of the SP system on the trivial solution curve $\{(0, \mu) \mid \mu \in \mathbb{R}\}$ during the iteration scheme. While the inner continuation is just the classical predictor-corrector continuation method for curve-tracking [22-26]. Starting with $\widetilde{\varphi}=0$, Equation (1.11a) is the governing equation for a BEC. Thus we can use an efficient predictor-corrector continuation algorithm [22-26] to compute the ground state solution of (1.11a). Next, we solve the Poisson equation (1.11c), and compute $(1.11 \mathrm{~d})$ to obtain $\widetilde{\varphi}$ for the next step continuation algorithm. Then we use the continuation algorithm again to compute the ground state solution of (1.11a). Here we need to evaluate the minimum eigenvalue of the Schrödinger eigenvalue problem. Similarly, we solve $(1.11 \mathrm{c})$ and compute $\widetilde{\varphi}$ for $(1.11 \mathrm{~d})$. We repeat the same process as above until two consecutive bifurcation points, say, $\mu_{k}$ and $\mu_{k+1}$, are close enough, which means the solution curves of the SP system obtained by the two-level continuation scheme will converge 
to the desired one. Then we can use the ground state solution obtained in the $k$ th outer continuation as an initial guess, and perform the (inexact) Newton iteration until it converges to the desired ground state solution.

This paper is organized as follows. In Section 2 we study spectral collocation methods (SCMs) for dipolar BEC and dipolar BEC in optical lattices in 3D. The two-level continuation scheme is described in Section 3 for computing the ground state solutions of Eq. (1.11). Section 4 shows that our numerical results are consistent with those in [13,20]. Moreover, numerical experiments for dipolar BEC with strongly anisotropic confining harmonic potential are presented. Finally, some concluding remarks are given in Section 5.

\section{SCMs for $3 \mathrm{D}$ dipolar BEC}

In this section, we will exploit the spectral accuracy of the SCMs in the context of numerical continuation methods for computing ground state solutions of 3D dipolar BEC, which is governed by the SP system (1.11a)-(1.11d). Here the first kind Chebyshev polynomials and the Fourier sine functions will be used as the basis functions for the trial function space. Since the macroscopic wave function $\phi(\mathbf{x}) \rightarrow 0$ rapidly as $|\mathbf{x}| \rightarrow \infty$, we replace the domain $\mathbb{R}^{3}$ in (1.11a)-(1.11d) by a sufficiently large domain $\Omega=(-L, L)^{3}$ with $L>0$ in our numerical computations. First we transform the domain $\Omega=(-L, L)^{3}$ into $\Omega_{1}=(-1,1)^{3}$. Using the change of variables $\mathbf{x} \rightarrow L \mathbf{x}$, Eqs. (1.11a)-(1.11d) are transformed into

$$
\begin{aligned}
& {\left[-\frac{1}{2 L^{2}} \Delta+\widetilde{V}(\mathbf{x})+(\beta-\lambda)|\phi(\mathbf{x})|^{2}-3 \lambda \widetilde{\varphi}(\mathbf{x})\right] \phi(\mathbf{x})=\mu \phi(\mathbf{x}), \mathbf{x} \in \Omega_{1}=(-1,1)^{3},} \\
& L^{3} \int_{\Omega_{1}}|\phi(\mathbf{x})|^{2} d \mathbf{x}=1, \\
& -\frac{1}{L^{2}} \Delta \varphi(\mathbf{x})=|\phi(\mathbf{x})|^{2}, \\
& \widetilde{\varphi}(\mathbf{x})=\frac{1}{L^{2}} \partial_{\mathbf{n n}} \varphi(\mathbf{x}),
\end{aligned}
$$

where $\widetilde{V}(\mathbf{x})=V(L \mathbf{x})$ and the boundary conditions are $\left.\phi\right|_{\partial \Omega_{1}}=0$ and $\left.\varphi\right|_{\partial \Omega_{1}}=0$.

Let $T_{n}(x)$ be the $n$th degree Chebyshev polynomial of the first kind, i.e., $T_{n}(x)=$ $\cos \left(n \cos ^{-1} x\right),-1 \leq x \leq 1$. Define the trial function space as

$$
S_{N}=\operatorname{span}\left\{p_{i}(x) p_{j}(y) p_{k}(z): i, j, k=0,1, \ldots, N-2\right\},
$$

where $p_{n}=T_{n}-T_{n+2}$. Then $\operatorname{dim} S_{N}=(N-1)^{3}$ and all the functions of $S_{N}$ satisfy the homogeneous Dirichlet boundary conditions. We choose $\left\{\left(x_{\ell}, y_{m}, z_{n}\right)=\left(\xi_{\ell}, \xi_{m}, \xi_{n}\right): \ell, m, n=\right.$ 
$0,1, \ldots, N-2\}$ as the collocation points, where $\xi_{i}=\cos \left(\frac{(2 i+1) \pi}{2(N-1)}\right), i=0,1, \ldots, N-2$, are the Chebyshev-Gauss points. To solve (2.1) using a two-level continuation scheme, first we let $\widetilde{\varphi}(\mathbf{x})=0$ in $(2.1 \mathrm{a})$, i.e., we solve the GPE

$$
\begin{aligned}
& {\left[-\frac{1}{2 L^{2}} \Delta+\widetilde{V}(\mathbf{x})+(\beta-\lambda)|\phi(\mathbf{x})|^{2}\right] \phi(\mathbf{x})=\mu \phi(\mathbf{x}), \mathbf{x} \in \Omega_{1},} \\
& \phi(\mathbf{x})=0, x \in \partial \Omega_{1} .
\end{aligned}
$$

The SCM for solving (2.3) is to find

$$
u_{N}(\mathbf{x})=\sum_{i, j, k=0}^{N-2} u_{i j k} p_{i}(x) p_{j}(y) p_{k}(z) \in S_{N}
$$

such that the residual function

$$
r_{1}(\mathbf{x})=\left[-\frac{1}{2 L^{2}} \Delta+\widetilde{V}(\mathbf{x})+(\beta-\lambda)\left|u_{N}(\mathbf{x})\right|^{2}\right] u_{N}(\mathbf{x})-\mu u_{N}(\mathbf{x})
$$

vanishes at the collocation points $\left(x_{\ell}, y_{m}, z_{n}\right)$. That is,

$$
\begin{aligned}
r_{1}\left(x_{\ell}, y_{m}, z_{n}\right)= & {\left[-\frac{1}{2 L^{2}} \Delta+\widetilde{V}\left(x_{\ell}, y_{m}, z_{n}\right)+(\beta-\lambda)\left|u_{N}\left(x_{\ell}, y_{m}, z_{n}\right)\right|^{2}\right] u_{N}\left(x_{\ell}, y_{m}, z_{n}\right) } \\
& -\mu u_{N}\left(x_{\ell}, y_{m}, z_{n}\right) \\
= & 0, \quad \ell, m, n=0,1, \ldots, N-2 .
\end{aligned}
$$

Denote $p_{i j}=p_{j}\left(x_{i}\right), p_{i j}^{\prime}=p_{j}^{\prime}\left(x_{i}\right), p_{i j}^{\prime \prime}=p_{j}^{\prime \prime}\left(x_{i}\right), v_{\ell m n}=\widetilde{V}\left(x_{\ell}, y_{m}, z_{n}\right)$, and define the $(N-$ $1) \times(N-1)$ matrices

$$
\begin{aligned}
& \mathbf{P}=\left[p_{i j}\right]_{0 \leq i, j \leq N-2}, \mathbf{P}^{\prime}=\left[p_{i j}^{\prime}\right]_{0 \leq i, j \leq N-2}, \mathbf{P}^{\prime \prime}=\left[p_{i j}^{\prime \prime}\right]_{0 \leq i, j \leq N-2}, \\
& \mathbf{U}^{(k)}=\left[u_{i j k}\right]_{0 \leq i, j \leq N-2}, k=0,1, \ldots, N-2, \\
& \mathbf{V}^{(n)}=\left[v_{\ell m n}\right]_{0 \leq \ell, m \leq N-2}, n=0,1, \ldots, N-2 .
\end{aligned}
$$

Let the vectors

$$
\widehat{\mathbf{U}}=\left[\begin{array}{c}
\operatorname{vec}\left(\mathbf{U}^{(0)}\right) \\
\operatorname{vec}\left(\mathbf{U}^{(1)}\right) \\
\vdots \\
\operatorname{vec}\left(\mathbf{U}^{(N-2)}\right)
\end{array}\right] \text { and } \widehat{\mathbf{V}}=\left[\begin{array}{c}
\operatorname{vec}\left(\mathbf{V}^{(0)}\right) \\
\operatorname{vec}\left(\mathbf{V}^{(1)}\right) \\
\vdots \\
\operatorname{vec}\left(\mathbf{V}^{(N-2)}\right)
\end{array}\right]
$$

where $\operatorname{vec}(\mathbf{M})$ denotes the vectorization of a matrix $\mathbf{M}$ formed by stacking the columns of $\mathbf{M}$ into a single column vector. Then $\widehat{\mathbf{U}}, \widehat{\mathbf{V}} \in \mathbb{R}^{(N-1)^{3}}$ and $(2.5)$ can be expressed as a generalized nonlinear eigenvalue problem

$$
H(\widehat{\mathbf{U}}, \mu)=-\frac{1}{2 L^{2}} \mathbf{A} \widehat{\mathbf{U}}+\widehat{\mathbf{V}} \circ(\mathbf{B} \widehat{\mathbf{U}})+(\beta-\lambda)(\mathbf{B} \widehat{\mathbf{U}})^{(3)}-\mu \mathbf{B} \widehat{\mathbf{U}}=0
$$


where "o" denotes the Hadamard product, $(\mathbf{B U})^{(3)}=(\mathbf{B U}) \circ(\mathbf{B U}) \circ(\mathbf{B U})$, and $\mathbf{A}, \mathbf{B} \in$ $\mathbb{R}^{(N-1)^{3} \times(N-1)^{3}}$ are defined by

$$
\begin{aligned}
& \mathbf{A}=\mathbf{P} \otimes \mathbf{P} \otimes \mathbf{P}^{\prime \prime}+\mathbf{P} \otimes \mathbf{P}^{\prime \prime} \otimes \mathbf{P}+\mathbf{P}^{\prime \prime} \otimes \mathbf{P} \otimes \mathbf{P} \\
& \mathbf{B}=\mathbf{P} \otimes \mathbf{P} \otimes \mathbf{P},
\end{aligned}
$$

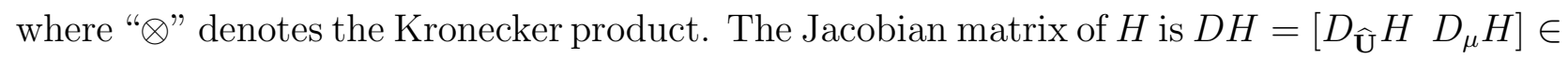
$\mathbb{R}^{(N-1)^{3} \times\left((N-1)^{3}+1\right)}$ with

$$
\begin{aligned}
& D_{\widehat{\mathbf{U}}} H=-\frac{1}{2 L^{2}} \mathbf{A} \widehat{\mathbf{U}}+\operatorname{diag}(\widehat{\mathbf{V}}) \mathbf{B}+3(\beta-\lambda) \operatorname{diag}\left((\mathbf{B} \widehat{\mathbf{U}})^{(2)}\right) \mathbf{B}-\mu \mathbf{B}, \\
& D_{\mu} H=-\mathbf{B} \widehat{\mathbf{U}}
\end{aligned}
$$

where $\operatorname{diag}(\mathbf{b})$ is a diagonal matrix with the vector $\mathbf{b}$ as the diagonal entries.

We use an efficient predictor-corrector continuation method to trace the ground state solution curve of (2.3) bifurcating at the first bifurcation point $\left(0, \mu_{1}\right)$. The constraint condition $(2.1 \mathrm{~b})$ is regarded as a stopping criterion for the curve-tracking [22]. We stop the first curve-tracking whenever the target point $\left(\phi_{1}^{*}, \mu_{1}^{*}\right)$ is reached for some chemical potential $\mu_{1}^{*}$ and $L^{3} \int_{\Omega_{1}}\left|\phi_{1}^{*}(\mathbf{x})\right|^{2} d \mathbf{x}=1$, where $\phi_{1}^{*}(\mathbf{x})=\sum_{i, j, k=0}^{N-1} u_{i j k}^{*} p_{i}(x) p_{j}(y) p_{k}(z)$.

Next, the spectral collocation formulation for solving (2.1c) is to find

$$
w_{N}(\mathbf{x})=\sum_{i, j, k=0}^{N-2} w_{i j k} p_{i}(x) p_{j}(y) p_{k}(z) \in S_{N}
$$

such that the residual function $r_{2}(\mathbf{x})=-\frac{1}{L^{2}} \Delta w_{N}(\mathbf{x})-\left|\phi_{1}^{*}(\mathbf{x})\right|^{2}$ vanishes at the collocation points $\left(x_{\ell}, y_{m}, z_{n}\right)$. That is,

$$
r_{2}\left(x_{\ell}, y_{m}, z_{n}\right)=-\frac{1}{L^{2}} \Delta w_{N}\left(x_{\ell}, y_{m}, z_{n}\right)-\left|\phi_{1}^{*}\left(x_{\ell}, y_{m}, z_{n}\right)\right|^{2}=0, \ell, m, n=0,1, \ldots, N-2 .
$$

Note that we also can use the fast Fourier transform (FFT) to solve (2.1c) which is more efficient than the SCM. Let $\widehat{\mathbf{W}}, \widehat{\mathbf{U}}^{*} \in \mathbb{R}^{(N-1)^{3}}$ be the column vectors corresponding to $w_{i j k}$ and $u_{i j k}^{*}$, respectively, as the definition of $\widehat{\mathbf{U}}$. We express $(2.11)$ as

$$
-\frac{1}{L^{2}} \mathbf{A} \widehat{\mathbf{W}}=\left(\mathbf{B} \widehat{\mathbf{U}}^{*}\right)^{2}
$$

where $\mathbf{A}$ and $\mathbf{B}$ are defined in (2.9). Denote the solution of (2.12) by the column vector $\widehat{\mathbf{W}}^{*}$ associated with $w_{i j k}^{*}$. The approximate solution of $(2.1 \mathrm{c})$ is $\varphi_{1}(\mathbf{x})=\sum_{i, j, k=0}^{N-2} w_{i j k}^{*} p_{i}(x) p_{j}(y) p_{k}(z)$. Then from $(2.1 \mathrm{~d})$ we obtain $\widetilde{\varphi}_{1}(\mathbf{x})=\frac{1}{L^{2}} \partial_{\mathbf{n n}} \varphi_{1}(\mathbf{x})$ for the next iterate of the continuation scheme. 
Now we consider $(2.1 \mathrm{a})$ with $\widetilde{\varphi}(\mathbf{x})=\widetilde{\varphi}_{1}(\mathbf{x}) \neq 0$. The SCM analogue of $(2.1 \mathrm{a})$ is

$$
F(\widehat{\mathbf{U}}, \mu)=-\frac{1}{2 L^{2}} \mathbf{A} \widehat{\mathbf{U}}+\widehat{\mathbf{V}} \circ(\mathbf{B} \widehat{\mathbf{U}})+(\beta-\lambda)(\mathbf{B} \widehat{\mathbf{U}})^{(3)}-3 \lambda \frac{1}{L^{2}}\left(\mathbf{C W}^{*}\right) \circ(\mathbf{B} \widehat{\mathbf{U}})-\mu \mathbf{B} \widehat{\mathbf{U}}=0,
$$

where $\mathbf{C} \in \mathbb{R}^{(N-1)^{3} \times(N-1)^{3}}$ is defined by

$$
\begin{aligned}
\mathbf{C}= & n_{1}^{2}\left(\mathbf{P} \otimes \mathbf{P} \otimes \mathbf{P}^{\prime \prime}\right)+n_{2}^{2}\left(\mathbf{P} \otimes \mathbf{P}^{\prime \prime} \otimes \mathbf{P}\right)+n_{3}^{2}\left(\mathbf{P}^{\prime \prime} \otimes \mathbf{P} \otimes \mathbf{P}\right) \\
& +2 n_{1} n_{2}\left(\mathbf{P} \otimes \mathbf{P}^{\prime} \otimes \mathbf{P}^{\prime}\right)+2 n_{2} n_{3}\left(\mathbf{P}^{\prime} \otimes \mathbf{P}^{\prime} \otimes \mathbf{P}\right)+2 n_{1} n_{3}\left(\mathbf{P}^{\prime} \otimes \mathbf{P} \otimes \mathbf{P}^{\prime}\right),
\end{aligned}
$$

and other symbols are defined as above. The Jacobian matrix of $F$ is $D F=\left[\begin{array}{ll}D_{\widehat{\mathbf{U}}} & D_{\mu} F\end{array}\right] \in$ $\mathbb{R}^{(N-1)^{3} \times\left((N-1)^{3}+1\right)}$ with

$$
\begin{aligned}
& D_{\widehat{\mathbf{U}}} F=D_{\widehat{\mathbf{U}}} H-3 \lambda \frac{1}{L^{2}} \operatorname{diag}\left(\mathbf{C}^{*}\right) \mathbf{B}, \\
& D_{\mu} F=-\mathbf{B} \widehat{\mathbf{U}},
\end{aligned}
$$

where $D_{\widehat{\mathbf{U}}} H$ is defined in (2.10). We use the FFT again to solve (2.1c), and compute $\widetilde{\varphi}(\mathbf{x})$ in $(2.1 \mathrm{~d})$ as above until $\widetilde{\varphi}(\mathbf{x})$ converges to a desired vector. Then the ground state solution of the dipolar BEC is the associated wave function $\phi(\mathbf{x})$.

For the 3D dipolar BEC in optical lattices, due to the high oscillation of periodic potential, we choose the Fourier sine functions as the basis functions for the SCM. See [25,26]. The trail function space using the Fourier sine functions as the basis functions is defined by

$$
\widetilde{S}_{N}=\operatorname{span}\left\{s_{i}(x) s_{j}(y) s_{k}(z): i, j, k=0,1, \ldots, N-2, x, y, z \in[-1,1]\right\},
$$

where $s_{n}(x)=\sin \left(\frac{(n+1) \pi(x+1)}{2}\right)$. Since $s_{n}( \pm 1)=0$, all the functions of $\widetilde{S}_{N}$ satisfy the homogeneous Dirichlet boundary conditions. In this case we must choose uniform grids on the domain $\Omega_{1}$ as the collocation points. The rest of the SCM using the Fourier sine functions as the basis functions is a straightforward modification of the first kind Chebyshev polynomials, and is omitted here.

\section{A two-level continuation scheme}

In numerical continuation methods, we use the energy level and wave function of the linear Schrödinger equation (LSE) as initial guesses to approximate the counterparts of the nonlinear GPE [22]. It is well known that Newton's method is widely used for solving nonlinear system of equations because its convergence rate is quadratic. However, an appropriate initial guess is, in general, not available for this method. The procedure described in Section 2 gives a heuristic idea to propose a two-level continuation scheme for tracing the first solution curves of the SP system (1.11), which can supply an appropriate initial guess for the 
Newton method to compute ground state solutions of dipolar BEC. In the first iterate of the outer continuation iteration we let $\widetilde{\varphi}(\mathbf{x})=\widetilde{\varphi}_{0}(\mathbf{x})=0$. The SCM analogue of (2.1a) is a parameter-dependent problem

$$
H(\widehat{\mathbf{U}}, \mu)=0,
$$

where $H: \mathbb{R}^{(N-1)^{3}} \times \mathbb{R} \rightarrow \mathbb{R}^{(N-1)^{3}}$ is defined in (2.8), which is a continuously differentiable function. A solution curve of (3.1) is denoted by

$$
\mathcal{C}_{H}=\{\mathbf{y}(s)=(\widehat{\mathbf{U}}(s), \mu(s)) \mid H(\widehat{\mathbf{U}}(s), \mu(s))=0, s \in I \subset \mathbb{R}\} .
$$

The bifurcation points of (3.1) on the trivial solution curve $\{(0, \mu) \mid \mu \in \mathbb{R}\}$ are just the eigenvalues of the LSE corresponding to (3.1). In order to compute the ground state solution of (3.1), we use a predictor-corrector continuation algorithm to trace the solution curve of (3.1) branching from the trivial solution curve at the first bifurcation point $\left(0, \mu_{1}\right)$, where $\mu_{1}$ is the minimum eigenvalue of the LSE. In addition, the normalization condition $(2.1 \mathrm{~b})$ is set as the target point for the curve-tracking. We start with a point $\mathbf{y}^{(0)}=\left(\widehat{\mathbf{U}}^{(0)}, \mu^{(0)}\right)=\left(0, \mu^{(0)}\right)$ on the trivial solution curve, which is close enough to the first bifurcation point. To compute the next approximating point $\mathbf{y}^{(1)}=\left(\widehat{\mathbf{U}}^{(1)}, \mu^{(1)}\right)$, we need to switch from the trivial solution curve to the nontrivial one. A well-known technique for branch-switching near the bifurcation point is to solve the perturbed problem

$$
H(\widehat{\mathbf{U}}, \mu)+\mathbf{d}=0
$$

where $\mathbf{d}$ is the perturbation vector which can be chosen as the eigenfunction of the LSE. Assume that we have successfully switched to the nontrivial solution curve. Let $\mathbf{y}^{(i)}=$ $\left(\widehat{\mathbf{U}}^{(i)}, \mu^{(i)}\right)$ be an accepted approximating point for the solution curve. In the $(i+1)$ th step of the continuation algorithm, we first compute the unit tangent vector $\dot{\mathbf{y}}^{(i)}=\left(\dot{\widehat{\mathbf{U}}}^{(i)}, \dot{\mu}^{(i)}\right)$ at $\mathbf{y}^{(i)}$ by solving the bordered linear system

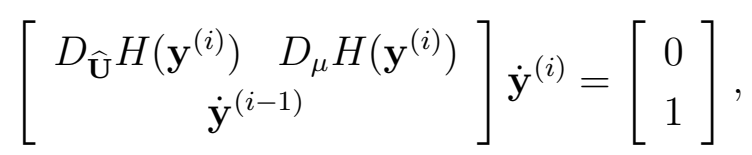

and a predicted point $\mathbf{z}^{(i+1,1)}$ of $\mathbf{y}^{(i+1)}$ can be obtained by the Euler predictor

$$
\mathbf{z}^{(i+1,1)}=\mathbf{y}^{(i)}+\delta_{i} \cdot \dot{\mathbf{y}}^{(i)},
$$

where $\delta_{i}>0$ is the step length which is determined by some step-size selection strategy. Next, we correct the predicted point $\mathbf{z}^{(i+1,1)}$ by performing Newton's iteration constrained to a hyperplane which is orthogonal to $\dot{\mathbf{y}}^{(i)}$. That is, we solve

$$
\left[\begin{array}{cc}
D_{\widehat{\mathbf{U}}} H\left(\mathbf{z}^{(i+1, j)}\right) & D_{\mu} H\left(\mathbf{z}^{(i+1, j)}\right) \\
\dot{\mathbf{y}}^{(i)}
\end{array}\right] \boldsymbol{\omega}^{(j)}=\left[\begin{array}{c}
-H\left(\mathbf{z}^{(i+1, j)}\right) \\
0
\end{array}\right], \quad j=1,2, \ldots,
$$


and set $\mathbf{z}^{(i+1, j+1)}=\mathbf{z}^{(i+1, j)}+\boldsymbol{\omega}^{(j)}, j=1,2, \ldots$. When $\left\|\boldsymbol{\omega}^{\left(j_{0}\right)}\right\|<\varepsilon$ and $\left\|H\left(\mathbf{z}^{\left(i+1, j_{0}+1\right)}\right)\right\|<\varepsilon$ for some $j_{0} \in \mathbb{N}$, where $\varepsilon>0$ is the accuracy for the Newton corrector, we finish the corrector step and set the next approximating point $\mathbf{y}^{(i+1)}=\mathbf{z}^{\left(i+1, j_{0}+1\right)}$. The first curve-tracking is implemented in this way until $\mathbf{y}^{\left(i_{0}\right)}=\left(\widehat{\mathbf{U}}^{\left(i_{0}\right)}, \mu^{\left(i_{0}\right)}\right)$ satisfies the normalization condition $L^{3} \int_{\Omega_{1}}\left|\sum_{i, j, k=0}^{N-1} u_{i j k}^{\left(i_{0}\right)} p_{i}(x) p_{j}(y) p_{k}(z)\right|^{2} d \mathbf{x}=1$ for some $i_{0} \in \mathbb{N}$. We set the target point $\mathbf{y}^{\left(i_{0}\right)}$ as $\left(\widehat{\mathbf{U}}^{*}, \mu_{1}^{*}\right)$, where the entries of $\widehat{\mathbf{U}}^{*}$, namely, $u_{i j k}^{*}$, are exploited to compute the ground state solution $\phi_{1}^{*}(\mathbf{x})=\sum_{i, j, k=0}^{N-1} u_{i j k}^{*} p_{i}(x) p_{j}(y) p_{k}(z)$.

Now we use $\left|\phi_{1}^{*}(\mathbf{x})\right|^{2}$ as the right-hand side of (2.1c) and solve the SCM analogue (2.12) to obtain the approximate solution $\varphi_{1}(\mathbf{x})$. Then we use $(2.1 \mathrm{~d})$ to compute $\widetilde{\varphi}_{1}(\mathbf{x})$ for the next iterate.

In the second iterate of the outer continuation iteration we let $\widetilde{\varphi}(\mathbf{x})=\widetilde{\varphi}_{1}(\mathbf{x})$. The SCM analogue of $(2.1 \mathrm{a})$ is a parameter-dependent problem of the form

$$
F(\widehat{\mathbf{U}}, \mu)=0,
$$

where $F: \mathbb{R}^{(N-1)^{3}} \times \mathbb{R} \rightarrow \mathbb{R}^{(N-1)^{3}}$ is defined in (2.13). To compute the ground state solution of (3.5), we use the predictor-corrector continuation algorithm to trace the solution curve of (3.5) branching the first bifurcation point $\left(0, \mu_{2}\right)$, where $\mu_{2}$ is the minimum eigenvalue of the LSE corresponding to (3.5). The normalization condition (2.1b) is regarded as the target point for the curve-tracking. We repeat the same process as the first iterate, and use (2.1c) and (2.1d) to obtain $\varphi_{2}(\mathbf{x})$ and $\widetilde{\varphi}_{2}(\mathbf{x})$. Similar procedures are executed until $\widetilde{\varphi}_{k}(\mathbf{x})$ converges. In practical computations we stop the curve-tracking if $\left\|\widetilde{\varphi}_{k}(\mathbf{x})-\widetilde{\varphi}_{k-1}(\mathbf{x})\right\|<\varepsilon_{1}$ for some number $\varepsilon_{1}>0$.

Our numerical experiments show that the above mentioned continuation scheme can effectively compute the ground states of a dipolar BEC and a dipolar BEC in optical lattices. However, curve-tracking for (2.1a) is time-consuming if the convergence behavior of $\left\{\widetilde{\varphi}_{k}(\mathbf{x})\right\}$ staggers. To overcome the drawback we propose a robust two-level continuation scheme as follows. If two consecutive bifurcation points of the ground state solution curves of (2.1a) are close enough, say, $\left|\mu_{k}-\mu_{k+1}\right|<\varepsilon_{2}$ for some $\varepsilon_{2}>0$, then the two solution curves will be close enough, too. Instead of tracing the solution curve bifurcating at $\left(0, \mu_{k+1}\right)$, we use the target point on the solution curve bifurcating at $\left(0, \mu_{k}\right)$ as the initial guess and perform the (inexact) Newton method to solve

$$
\widetilde{F}(\widehat{\mathbf{U}}, \mu)=\left[\begin{array}{l}
F(\widehat{\mathbf{U}}, \mu) \\
F_{1}(\widehat{\mathbf{U}}, \mu)
\end{array}\right]=0,
$$

where $F$ is the same as above and $F_{1}: \mathbb{R}^{(N-1)^{3}} \times \mathbb{R} \rightarrow \mathbb{R}$ is the normalization constraint 
which is defined by $F_{1}(\widehat{\mathbf{U}}, \mu)=L^{3} \int_{\Omega_{1}}\left|\sum_{i, j, k=0}^{N-1} u_{i j k} p_{i}(x) p_{j}(y) p_{k}(z)\right|^{2} d \mathbf{x}-1$. That is, we solve (2.1a) and (2.1b) simultaneously. We set the solution by $\left(\phi_{k, 1}^{*}(\mathbf{x}), \mu_{k, 1}^{*}\right)$ and then use (2.1c) and (2.1d) to obtain $\varphi_{k, 1}(\mathbf{x})$ and $\widetilde{\varphi}_{k, 1}(\mathbf{x})$. The function $\widetilde{\varphi}_{k, 1}(\mathbf{x})$ will be used as the initial guess for the (inexact) Newton method. We solve (3.6) iteratively until the function $\widetilde{\varphi}_{k, \ell}(\mathbf{x})$ converges to the desired ground state solution $\left(\phi^{*}(\mathbf{x}), \mu^{*}\right)$. The detailed procedure of the two-level continuation scheme for computing the ground state of a dipolar BEC is described as follows.

\section{Algorithm 3.1 The two-level continuation scheme for computing energy levels of a dipolar BEC.}

Input:

$k:=$ The $k$ th iterate of the iterative continuation scheme.

$K:=$ Maximum number of the outer continuation iteration.

$\varepsilon:=$ Accuracy tolerance for approximating points on each solution curve.

$\varepsilon_{1}:=$ Accuracy tolerance for $\widetilde{\varphi}_{k, \ell}$.

$\varepsilon_{2}:=$ Accuracy tolerance for two consecutive bifurcation points.

$\widetilde{\varphi}_{0}:=0, k:=0$.

Outer continuation iteration:

Step 1: (i) $k=k+1$.

(ii) If $k=K$, stop.

(iii) Inner continuation iteration: use the predictor-corrector continuation algorithm to trace the ground state solution curve of (2.1a) until the target point $\left(\phi_{k}^{*}, \mu_{k}^{*}\right)$ is reached.

Step 2: Use the Fast Fourier Transform (FFT) to solve (2.1c) for $\varphi_{k}$.

Step 3: Use (2.1d) to compute $\widetilde{\varphi}_{k}$.

Step 4: Check for convergence:

(i) Compute the first bifurcation point $\left(0, \mu_{k+1}\right)$ of (2.1a) with $\widetilde{\varphi}=\widetilde{\varphi}_{k}$.

(ii) if $\left|\mu_{k}-\mu_{k+1}\right|>\varepsilon_{2}$,

go to Step 1.

else

(a) Set $\ell=0$ and $\left(\phi_{k, 0}^{*}, \mu_{k, 0}^{*}\right)=\left(\phi_{k}^{*}, \mu_{k}^{*}\right), \widetilde{\varphi}_{k, 0}=\widetilde{\varphi}_{k}$.

(b) $\ell=\ell+1$.

(c) Use the approximate solution $\left(\phi_{k, \ell-1}^{*}, \mu_{k, \ell-1}^{*}\right)$ as the initial guess, and perform the (inexact) Newton method to solve (2.1a) and (2.1b) simultaneously.

(d) Set the approximate solution obtained in (c) by $\left(\phi_{k, \ell}^{*}, \mu_{k, \ell}^{*}\right)$. 
(e) Use the FFT to solve (2.1c) for $\varphi_{k, \ell}$.

(f) Use (2.1d) to compute $\widetilde{\varphi}_{k, \ell}$.

(g) Repeat the procedure (b)-(f) until $\left\|\widetilde{\varphi}_{k, \ell}-\widetilde{\varphi}_{k, \ell-1}\right\|<\varepsilon_{1}$, and the desired solution is $\left(\phi^{*}, \mu^{*}\right)$.

end if.

(iii) exit.

In order to efficiently implement Algorithm 3.1, the parameter $\varepsilon_{2}$ must be properly chosen with care. Since the inner continuation in Step 1 is time-consuming, the parameter $\varepsilon_{2}$ should be large enough so that we can omit unnecessary procedure for curve-tracking. On the other hand, $\varepsilon_{2}$ must be small enough so that the two consecutive target points $\left(\phi_{k-1}^{*}, \mu_{k-1}^{*}\right)$ and $\left(\phi_{k}^{*}, \mu_{k}^{*}\right)$ will be close enough, which guarantees that the Newton method will converge.

Note that the minimum eigenvalue of the LSE associated with the governing GPE for dipolar BEC is simple. In addition, the discrete matrix corresponding to the Jacobian matrix $D_{\widehat{\mathbf{U}}} H$ (or $D_{\widehat{\mathbf{U}}} F$ ) is nonsymmetric. Therefore, it is quite easy to compute the minimum eigenvalue of the LSE. The detection of multiple eigenvalues in a nonlinear eigenvalue problem using numerical continuation methods combined with linear solvers may go back to the eighties of last century. When the Fréchet derivative $D_{\widehat{\mathbf{U}}} H\left(\right.$ or $D_{\widehat{\mathbf{U}}} F$ ) is symmetric, one can either use the direct linear solver, e.g., the Gaussian elimination [27] or the iterative linear solver, e.g., the block Lanczos method $[28,29]$ to solve linear system of equations and to detect multiplicity of an eigenvalue in curve-tracing. On the other hand, when the Fréchet derivative $D_{\widehat{\mathbf{U}}} H$ (or $D_{\widehat{\mathbf{U}}} F$ ) is nonsymmetric, one can use the Arnoldi method [30,31] to solve linear systems and so on.

\section{Numerical results}

The two-level continuation scheme described in Algorithm 3.1 was implemented to compute the ground state solutions of 3D dipolar BEC and dipolar BEC in optical lattices, where the predictor-corrector continuation method was used to trace the ground state solution curves of the GPE. The accuracy tolerance for the (inexact) Newton corrector and $\widetilde{\varphi}_{k, \ell}$ are $\varepsilon=5 \times 10^{-8}$ and $\varepsilon_{1}=5 \times 10^{-9}$, respectively. In Examples $1-2$ and $4-5$ we used the first kind Chebyshev polynomials with $N=26$ as the basis functions to discretize the SP system. But in Example 3 we chose the Fourier sine functions with $N=32$ as the basis functions because of the high oscillation of the periodic potential. Note that the numerical experiments in Examples 4-5 have never been reported in published literatures. All computations were executed in the domain $\Omega=(-8,8)^{3}$ on an Intel $^{\circledR}$ Core $^{\mathrm{TM}}$ i7-2600K PC using MATLAB 
language. The following notations are used in Tables 1-4.

$\begin{aligned} & \text { NOMENCLATURE } \\ & E \text { : } \text { the total energy of the ground state solution of dipolar BEC. } \\ & E_{\text {kin }}, E_{\text {pot }}, E_{\text {int }}, E_{\text {dip }}: \text { various energies of the ground state solution of dipolar BEC (defined } \\ &\text { in }(1.9)) . \\ & \sigma_{x}, \sigma_{y}, \sigma_{z}: \text { the condensate widths which are defined as } \sigma_{\alpha}=\int_{\mathbb{R}^{3}} \alpha^{2}|\phi(\mathbf{x})|^{2} d \mathbf{x}, \\ & \alpha=x, y, z . \\ & \rho(\mathbf{0}): \text { the central density which is defined as } \rho(\mathbf{0})=|\phi(0,0,0)|^{2} . \\ & \mu_{k}, k=1,2, \ldots: \text { the first bifurcation point of each curve of dipolar BEC. } \\ & \mu_{k}^{*}, k=1,2, \ldots: \text { the energy level of the approximate ground state solution of the } k \text { th } \\ & \text { iterate solution curve. } \\ & \mu_{k, \ell}^{*}, \ell=1,2, \ldots: \text { the energy level of the approximate ground state solution obtained } \text { by implementing the (inexact) Newton method. } \\ & \mu^{*}: \text { the energy level of the ground state solution of the dipolar BEC. }\end{aligned}$

Example 1 (Comparisons between Algorithm 3.1 and the backward Euler sine pseudospectral method). We used the first kind Chebyshev polynomials as the basis functions combined with the two-level continuation scheme to compute the ground state solution of (1.11), where $V(\mathbf{x})=\left(x^{2}+y^{2}+0.25 z^{2}\right) / 2, \mathbf{n}=(0,0,1)^{T}$, and $\beta=207.16$. These data are the same as those used in [13]. Table 1 lists various types of energy quantities for the ground state solutions of dipolar BEC with respect to different $\lambda$ values obtained by implementing Algorithm 3.1 (first row) and the backward Euler sine pseudospectral method in [13] (second row), where we used the Gaussian quadrature formula to approximate the energy quantities in (1.9). From Table 1 we see that the energy quantities obtained by these two different numerical methods are at least consistent within the second decimal digit. Note that the accuracy tolerance used in [13] is $10^{-6}$, whereas we used $5 \times 10^{-9}$. Figure 1 shows the isosurface plots of $|\phi(\mathbf{x})|=0.01$ for the ground state solution of (1.11) with $\lambda=-0.5 \beta,-0.25 \beta, 0.0 \beta$, and $0.25 \beta$. For $\lambda= \pm 0.25 \beta$ and $0.0 \beta$, we only need to trace the solution curves once, where the coefficient of the cubic nonlinearity $\beta-\lambda \in(155,259)$. That is, we can efficiently compute the ground state solutions when there is no long-range dipolar interaction in the system, or the coefficients of the cubic nonlinear term as well as the dipolar term are small compared to the other data used in our numerical experiments.

Example 2 ( $3 D$ dipolar BEC with the harmonic potential and double-well potential). In this example we also chose the same data as those used in [13], namely, $\beta=401.432, \lambda=$ 64.22912, the dipolar direction $\mathbf{n}=(0,0,1)^{T}$, the harmonic potential $V(\mathbf{x})=\left(x^{2}+y^{2}+z^{2}\right) / 2$, and the double-well potential $V(\mathbf{x})=\left(x^{2}+y^{2}+z^{2}\right) / 2+4 e^{-z^{2} / 2}$. In addition, the accuracy 
for two consecutive bifurcation points is $\varepsilon_{2}=0.2$. Table 2 lists the implementation details of Algorithm 3.1, i.e., the minimum eigenvalues of the SEP, and associated energy levels of the ground state solutions, and the convergence behavior of the (inexact) Newton iteration. Figures 2(a1)-2(a2) show that for both the harmonic potential and double-well potential we only need to trace the first solution curves twice during the implementation of Algorithm 3.1. Figures 2(b1)-2(c1) and 2(b2)-2(c2) show that the contour plots of $|\phi(x, 0, z)|^{2}$ and the isosurface plots of $|\phi(\mathbf{x})|=0.01$ for the ground state solutions are identical with those given in [13]. Note that in the case of double-well potential, the isosurface plot of 3D dipolar BEC is dumb-bell shaped, which shows that the shape is determined by the type of potential we choose.

Example 3 ( $3 D$ dipolar BEC in optical lattices). For comparison we chose the same data as those in [13], namely, $V(\mathbf{x})=\left(x^{2}+y^{2}+z^{2}\right) / 2+100\left(\sin ^{2}(\pi x / 2)+\sin ^{2}(\pi y / 2)+\sin ^{2}(\pi z / 2)\right)$, $\beta=401.432, \lambda=64.22912$, and $\mathbf{n}=(0,0,1)^{T}$. The accuracy tolerance for two consecutive bifurcation points is $\varepsilon_{2}=0.7$. Because of the high oscillation of the periodic potential, we used the Fourier sine functions as the basis functions. Table 3 lists the implementation details of Algorithm 3.1, i.e., the minimum eigenvalues of the SEP, and associated energy levels of the ground state solutions during the outer continuation process, together with the convergence behavior of the (inexact) Newton method. Figure 3(a) shows that we only need to trace the first solution curve twice to compute the ground state solution. The contour plot of $|\phi(x, 0, z)|^{2}$ and the isosurface plot of $|\phi(\mathbf{x})|=0.04$ displayed in Figures 3(b) and $3(\mathrm{c})$, respectively, are more delicate than the counterparts shown in [13], which show how the optical lattices would affect the structure of 3D dipolar BEC.

Example 4 (3D dipolar BEC with various choices of dipolar directions). (i) Consider (1.11) with $V(\mathbf{x})=\left(x^{2}+y^{2}+z^{2}\right) / 2, \beta=207.16$, and $\lambda=-103.58$. We chose three different dipolar directions $\mathbf{n}=(0,0,1)^{T}, \mathbf{n}=(0,1,0)^{T}$, and $\mathbf{n}=(1,1,1)^{T} / \sqrt{3}$. Figure 4 shows the contour plots of $|\phi(0, y, z)|^{2},|\phi(x, y, 0)|^{2}$, and the isosurface plots of $|\phi(\mathbf{x})|=0.08$ for the ground state of the dipolar BEC with different dipolar directions. In particular, Figures 4(a1) and 4(b1) show that the contour plots of $|\phi(0, y, z)|^{2}$ are ellipse with long axis parallel to the $y$-axis and $z$-axis, respectively, where the dipole components on these axes are zero. When the dipole axis has equal weight on each component, Figure 4(c1) shows that the contour plot of $|\phi(0, y, z)|^{2}$ is also an ellipse with the long axis lying on the line $y=-z$, and forms an angle of 45 degree with the $(y, z)$-plane. Note that the contour plots displayed in Figures $4(\mathrm{c} 1)$ and $4(\mathrm{c} 2)$ are the same because the dipole axis has equal weight on each direction. The condensate widths $\sigma_{x}, \sigma_{y}, \sigma_{z}$ in the $x$-, $y$-, and $z$-direction, respectively, are listed in Table 4(a). 
(ii) Consider (1.11) with $V(\mathbf{x})=\left(x^{2}+y^{2}+z^{2}\right) / 2, \beta=207.16$, and $\lambda=51.79$. We chose four different dipolar directions $\mathbf{n}=(0,0,1)^{T}, \mathbf{n}=(0,1,1)^{T} / \sqrt{2}, \mathbf{n}=(1,1,0)^{T} / \sqrt{2}$, and $\mathbf{n}=(1,2,1)^{T} / \sqrt{6}$. Figure 5 shows the contour plots of $|\phi(0, y, z)|^{2},|\phi(x, y, 0)|^{2}$, and the isosurface plots of $|\phi(\mathbf{x})|=0.08$ for the ground state of the dipolar BEC with different dipolar directions. Figure 5(a1) shows that the contour plot of $|\phi(0, y, z)|^{2}$ is an ellipse with long axis parallel to the $z$-axis. By comparing to the contour plot of Figure 4(a1) we observe that the coefficient $\beta-\lambda$ of the cubic nonlinear term could affect the shape of the 3D dipolar BEC. See the difference between Figures 4(a3) and 5(a3). It is straightforward to see that the contour plots displayed in Figures 5(b1) and 5(c2) are the same because of the dipole axis and the surfaces we choose. Note that the long axis of the ellipse shown in Figures $5(\mathrm{~d} 1)$ and ( $\mathrm{d} 2$ ) lies on the line $y=2 z$ and $y=2 x$, respectively, because the weight of the dipole axis on the $y$-component is greater than those on the $z$ - and $x$-component. The condensate widths $\sigma_{x}, \sigma_{y}, \sigma_{z}$ in the $x$-, $y$-, and $z$-direction, respectively, are listed in Table 4(b).

Example 5 (Pancake-shaped and cigar-shaped dipolar BEC). (i) When the condensates are confined by a strong harmonic trap in the $z$-direction, the BEC shape will typically like a pancake. Here we chose $V(\mathbf{x})=\left(x^{2}+y^{2}+25 z^{2}\right) / 2, \mathbf{n}=(0,0,1)^{T}, \beta=207.16$ and $\lambda=51.79$ in (1.11), and $\varepsilon_{2}=0.7$ in Algorithm 3.1. Figure 6(a) shows the solution curves of each outer continuation iterate, where the bifurcation points $\mu_{1}=2.48271, \mu_{2}=7.17415, \mu_{3}=5.82802$, $\mu_{4}=6.46679$, and we need to trace the first solution curves three times. The energy level of the ground state solution is $\mu^{*}=10.67684$. The isosurface plot of $|\phi(\mathbf{x})|=0.05$ for the ground state solution is shown in Figure 6(b), where the shape is oblate. For comparison we chose $\varepsilon_{2}=0.4$. Figure 6 (c) shows that we need to trace the first solution curves four times, which requires more execution time than the former.

(ii-a) When the condensates are confined by a strong harmonic trap in the $(y, z)$-plane, the BEC shape will typically be prolate like a cigar. We chose $V(\mathbf{x})=\left(x^{2}+25 y^{2}+25 z^{2}\right) / 2$, $\mathbf{n}=(0,0,1)^{T}, \beta=207.16$ and $\lambda=51.79$ in (1.11), and $\varepsilon_{2}=1.8$ in Algorithm 3.1. Figure 7 (a) shows that we need to trace the solution curves three times, where the bifurcation points $\mu_{1}=3.46633, \mu_{2}=10.20626, \mu_{3}=7.31243, \mu_{4}=9.07596$, and the energy level of the ground state solution is $\mu^{*}=18.63855$. Figure $7(\mathrm{~b})$ shows the isosurface plot of $|\phi(\mathbf{x})|=0.05$ for the ground state solution, where the dipole axis is perpendicular to the cigar-shaped direction. For comparison we also chose $\varepsilon_{2}=0.4$. Figure $7(\mathrm{c})$ shows that we need to trace the first solution curves six times, which is much more expensive than the former.

(ii-b) When the condensates are confined by a strong harmonic trap in the $(x, y)$-plane, the BEC shape will typically be prolate like a cigar. We chose $V(\mathbf{x})=\left(25 x^{2}+25 y^{2}+z^{2}\right) / 2$, $\mathbf{n}=(0,0,1)^{T}, \beta=207.16$ and $\lambda=51.79$ in (1.11), and $\varepsilon_{2}=0.2$ in Algorithm 3.1. Figure 
8(a) shows that we only need to trace the solution curves twice, where the bifurcation points $\mu_{1}=3.46633, \mu_{2}=4.28289, \mu_{3}=4.17300$, and the energy level of the ground state solution is $\mu^{*}=16.96916$. Figure 8 (b) shows the isosurface plot of $|\phi(\mathbf{x})|=0.05$ for the ground state solution, where the dipole axis is parallel to the cigar-shaped direction.

\section{Conclusions}

We have described the SCMs combined with the two-level continuation scheme for computing the ground state solutions of the Schrödinger-Poisson system, where the first kind Chebyshev polynomials and the Fourier sine functions were used as the basis functions for the trial function space. The two-level continuation scheme combined with Fourier sine collocation method supplies accurate numerical solutions for dipolar BEC and dipolar BEC in optical lattices. The numerical results of Examples 1-3 are identical with those reported in [13]. In addition, the results of Example 3 are more delicate than the counterparts in [13]. Based on the numerical results reported in Section 4, we wish to give some concluding remarks concerning the performance of Algorithm 3.1. In particular, we will discuss the physical consequences of Examples 4-5 which are not reported in [13].

(i) The SCMs combined with Algorithm 3.1 can efficiently compute accurate ground state solutions for 3D dipolar BEC when the harmonic trapping potential is isotropic, i.e., $\gamma_{x}^{2}=\gamma_{y}^{2}=\gamma_{z}^{2}$, and is independent of the basis functions we use. However, when the periodic potential is imposed on the GPE, it would be better to use the Fourier sine functions as the basis functions rather than to use the Chebyshev polynomials or even the Legendre polynomials. This is because the collocation points of the former locate uniformly on the domain, while most collocation points of the latter locate uniformly near the boundary, which can not fit the high oscillation of optical lattices.

(ii) In Examples 1-4 we only need to trace the first solution curves of the SP system once or twice to compute the ground state solutions of 3D dipolar BEC and 3D dipolar BEC in optical lattices. On the other hand, in Example 5 we need to trace the solution curves either twice or three times. The results show that the cost of computing the ground state solutions of 3D dipolar BEC is independent of the type of trapping potential we choose, but more or less depends on whether the GPE is quasi-1D or quasi-2D.

(iii) From the numerical results of Example 5 we observe that when the condensates are confined by a strong harmonic trap in the $z$-direction, i.e., the trap frequency in the $z$-direction is much greater than those in the $x$ - and $y$-direction, say, $\omega_{x}^{2}=\omega_{y}^{2} \ll \omega_{z}^{2}$, 
then the governing equation for the 3D dipolar BEC degenerates to a quasi-2D GPE. Thus the shape of dipolar BEC is confined on the $(x, y)$-plane, and looks like a pancake. See Figure 6(b). Analogously, when the condensates are confined by a strong harmonic trap in the $(y, z)$-plane, i.e., $\omega_{x}^{2} \ll \omega_{y}^{2}=\omega_{z}^{2}$, then the governing equation for the $3 \mathrm{D}$ dipolar BEC degenerates to a quasi-1D GPE. Thus the shape of dipolar BEC looks like a cigar which is parallel to the $x$-direction and perpendicular to the $(y, z)$-plane. See Figure 7(b). Similarly, when the condensates are confined by a strong harmonic trap in the $(x, y)$-plane, then the shape of dipolar BEC also looks like a cigar, and is parallel to the $z$-direction. We conclude that the numerical results of Example 5 are consistent with the theoretic prediction of dipolar BEC [20,32].

\section{Acknowledgments}

The authors would like to thank the two referees for their valuable comments that have improved the original version of this paper. 
Table 1: Different quantities of the ground state solution of dipolar BEC obtained using Algorithm 3.1 (first row) and the backward Euler sine pseudospectral method (second row), where $V(x, y, z)=\left(x^{2}+y^{2}+0.25 z^{2}\right) / 2, \mathbf{n}=(0,0,1)^{T}$, and $\beta=207.16$.

\begin{tabular}{cllllllllc}
\hline$\lambda$ & $\mu^{*}$ & $E$ & $E_{\text {kin }}$ & $E_{\text {pot }}$ & $E_{\text {int }}$ & $E_{\text {dip }}$ & $\sigma_{x}$ & $\sigma_{z}$ & $\rho(\mathbf{0})$ \\
\hline \multirow{2}{*}{$-0.5 \beta$} & 3.9265 & 2.9568 & 0.2653 & 1.7218 & 0.8376 & 0.1321 & 1.1516 & 1.7789 & 0.01568 \\
& 3.927 & 2.957 & 0.265 & 1.721 & 0.839 & 0.131 & 1.153 & 1.770 & 0.01575 \\
\hline \multirow{2}{*}{$0.25 \beta$} & 3.8154 & 2.8832 & 0.2743 & 1.6768 & 0.8513 & 0.0809 & 1.1103 & 1.8846 & 0.01600 \\
& 3.817 & 2.883 & 0.274 & 1.675 & 0.853 & 0.081 & 1.111 & 1.879 & 0.01605 \\
\hline \multirow{2}{*}{$0.0 \beta$} & 3.6821 & 2.7946 & 0.2860 & 1.6211 & 0.8875 & 0.0000 & 1.0667 & 1.9661 & 0.01689 \\
& 3.684 & 2.794 & 0.286 & 1.618 & 0.890 & 0.000 & 1.066 & 1.962 & 0.01693 \\
\hline \multirow{2}{*}{$0.25 \beta$} & 3.5239 & 2.6894 & 0.3022 & 1.5528 & 0.9488 & -0.1144 & 1.0176 & 2.0343 & 0.01843 \\
& 3.525 & 2.689 & 0.303 & 1.550 & 0.950 & -0.114 & 1.017 & 2.030 & 0.01842 \\
\hline
\end{tabular}


Table 2: The first bifurcation points and associated energy levels of (1.11) with various choices of potentials, where $\mathbf{n}=(0,0,1)^{T}, \beta=401.432$, and $\lambda=64.22912$.

(a) The harmonic potential $V(x, y, z)=\left(x^{2}+y^{2}+z^{2}\right) / 2$

\begin{tabular}{|c|c|c|c|c|c|c|}
\hline$k$ & $\mu_{k}$ & $\left|\mu_{k}-\mu_{k-1}\right|$ & $\mu_{k}^{*}$ & $\ell$ & $\mu_{2, \ell}^{*}$ & $\left\|\widetilde{\varphi}_{2, \ell}-\widetilde{\varphi}_{2, \ell-1}\right\|$ \\
\hline 1 & 1.499103608 & - & 5.759102362 & 1 & 6.113461748 & $2.59 \mathrm{e}-05$ \\
\hline 2 & 2.404863787 & 0.905760179 & 6.164862372 & 2 & 6.109090403 & $1.18 \mathrm{e}-06$ \\
\hline \multirow[t]{7}{*}{3} & 2.243135579 & 0.161728208 & - & 3 & 6.108440777 & $4.12 \mathrm{e}-07$ \\
\hline & & & & 4 & 6.108654507 & $1.47 \mathrm{e}-07$ \\
\hline & & & & 5 & 6.108585971 & $5.34 \mathrm{e}-08$ \\
\hline & & & & 6 & 6.108608817 & $1.97 \mathrm{e}-08$ \\
\hline & & & & 7 & 6.108600976 & $7.36 \mathrm{e}-09$ \\
\hline & & & & 8 & 6.108603738 & $2.77 \mathrm{e}-09$ \\
\hline & & & & & $=6.108603738$ & \\
\hline
\end{tabular}

(b) The double-well potential $V(x, y, z)=\left(x^{2}+y^{2}+z^{2}\right) / 2+4 e^{-z^{2} / 2}$

\begin{tabular}{|c|c|c|c|c|c|c|}
\hline$k$ & $\mu_{k}$ & $\left|\mu_{k}-\mu_{k-1}\right|$ & $\mu_{k}^{*}$ & $\ell$ & $\mu_{2, \ell}^{*}$ & $\left\|\widetilde{\varphi}_{2, \ell}-\widetilde{\varphi}_{2, \ell-1}\right\|$ \\
\hline 1 & 4.097792572 & - & 7.557791027 & 1 & 7.798126857 & $8.12 \mathrm{e}-06$ \\
\hline 2 & 4.665386406 & 0.567593834 & 7.825384750 & 2 & 7.798651607 & $2.38 \mathrm{e}-07$ \\
\hline \multirow[t]{5}{*}{3} & \multirow[t]{5}{*}{4.596890388} & \multirow[t]{5}{*}{0.068496018} & \multirow[t]{5}{*}{ - } & 3 & 7.797968763 & $6.07 \mathrm{e}-08$ \\
\hline & & & & 4 & 7.798213430 & $2.29 \mathrm{e}-08$ \\
\hline & & & & 5 & 7.798124361 & $8.64 \mathrm{e}-09$ \\
\hline & & & & 6 & 7.798157185 & $3.25 \mathrm{e}-09$ \\
\hline & & & & \multicolumn{3}{|c|}{$\mu^{*}=7.798157185$} \\
\hline
\end{tabular}


Table 3: The first bifurcation points and associated energy levels of (1.11) with periodic potential $V(x, y, z)=\left(x^{2}+y^{2}+z^{2}\right) / 2+100\left(\sin ^{2}(\pi x / 2)+\sin ^{2}(\pi y / 2)+\sin ^{2}(\pi z / 2)\right)$, where $\mathbf{n}=(0,0,1)^{T}, \beta=401.432$, and $\lambda=64.22912$.

\begin{tabular}{|c|c|c|c|c|c|c|}
\hline$k$ & $\mu_{k}$ & $\left|\mu_{k}-\mu_{k-1}\right|$ & $\mu_{k}^{*}$ & $\ell$ & $\mu_{2, \ell}^{*}$ & $\left\|\widetilde{\varphi}_{2, \ell}-\widetilde{\varphi}_{2, \ell-1}\right\|$ \\
\hline 1 & 17.761137069 & - & 44.921067638 & 1 & 46.766377910 & $4.30 \mathrm{e}-05$ \\
\hline 2 & 23.171227052 & 5.410089983 & 46.831148476 & 2 & 46.759383322 & $1.59 \mathrm{e}-05$ \\
\hline \multirow[t]{6}{*}{3} & 22.508523176 & 0.662703876 & - & 3 & 46.759219086 & $2.94 \mathrm{e}-06$ \\
\hline & & & & 4 & 46.759259694 & $5.51 \mathrm{e}-07$ \\
\hline & & & & 5 & 46.759252498 & $1.03 \mathrm{e}-07$ \\
\hline & & & & 6 & 46.759253758 & $1.94 \mathrm{e}-08$ \\
\hline & & & & 7 & 46.759253536 & $3.66 \mathrm{e}-09$ \\
\hline & & & & & $=46.759253536$ & \\
\hline
\end{tabular}

Table 4: The condensate widths of dipolar BECs with $V(\mathbf{x})=\left(x^{2}+y^{2}+z^{2}\right) / 2, \beta=207.16$, and different values of $\lambda$ and $\mathbf{n}$.

(a) $\lambda=-103.58$

\begin{tabular}{cccc}
\hline $\mathbf{n}$ & $\sigma_{x}$ & $\sigma_{y}$ & $\sigma_{z}$ \\
\hline$(0,0,1)^{T}$ & 1.26742 & 1.26742 & 0.94056 \\
$(0,1,0)^{T}$ & 1.26742 & 0.94056 & 1.26742 \\
$(1,1,1)^{T} / \sqrt{3}$ & 1.16862 & 1.16862 & 1.16862 \\
\hline
\end{tabular}

(b) $\lambda=51.79$

\begin{tabular}{cccc}
\hline $\mathbf{n}$ & $\sigma_{x}$ & $\sigma_{y}$ & $\sigma_{z}$ \\
\hline$(0,0,1)^{T}$ & 1.13586 & 1.13586 & 1.26454 \\
$(0,1,1)^{T} / \sqrt{2}$ & 1.13596 & 1.20143 & 1.20143 \\
$(1,1,0)^{T} / \sqrt{2}$ & 1.20143 & 1.20143 & 1.13596 \\
$(1,2,1)^{T} / \sqrt{6}$ & 1.15820 & 1.22259 & 1.15820 \\
\hline
\end{tabular}




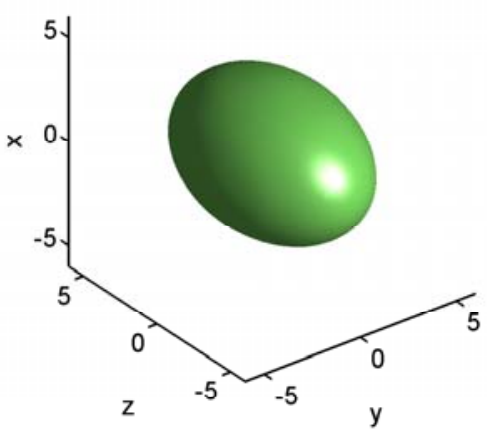

(a) $\lambda=-0.5 \beta$

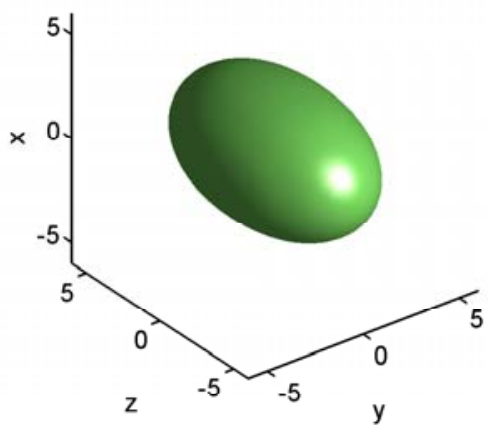

(c) $\lambda=0.0 \beta$

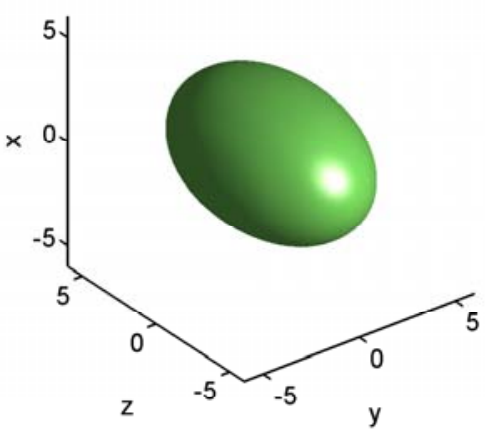

(b) $\lambda=-0.25 \beta$

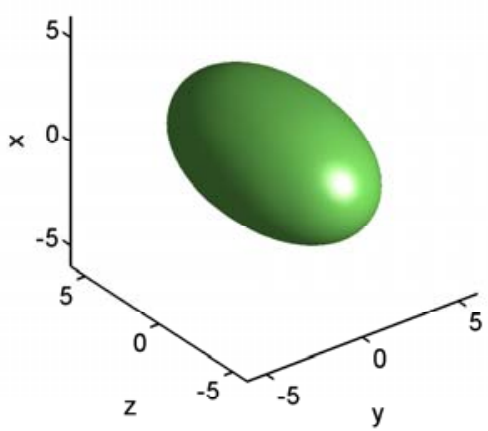

(d) $\lambda=0.25 \beta$

Figure 1: The isosurface plots of $|\phi(\mathbf{x})|=0.01$ for the ground state solution of (1.11), where $V(\mathbf{x})=\left(x^{2}+y^{2}+0.25 z^{2}\right) / 2, \mathbf{n}=(0,0,1)^{T}, \beta=207.16$, and different values of $\lambda$. 


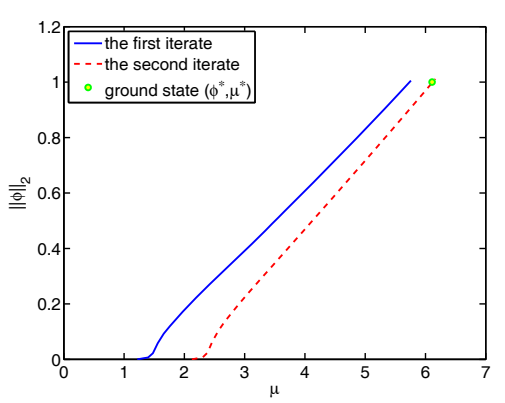

(a1) solution curves

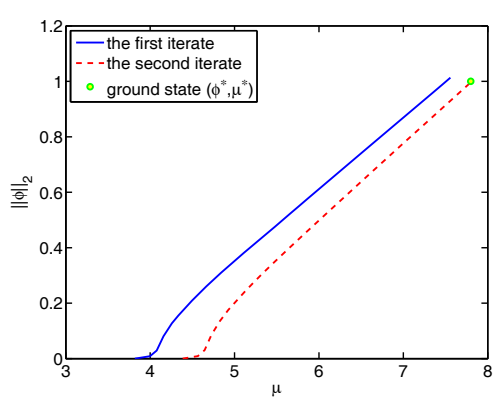

(a2) solution curves

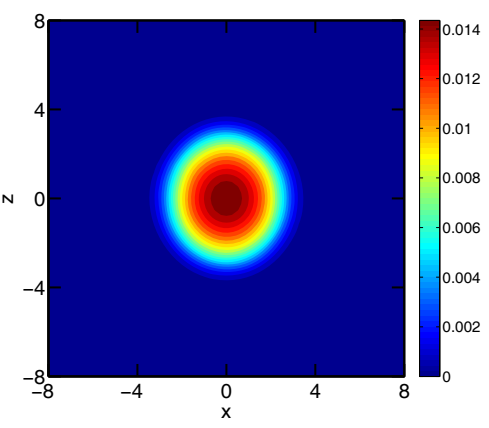

(b1) $|\phi(x, 0, z)|^{2}$

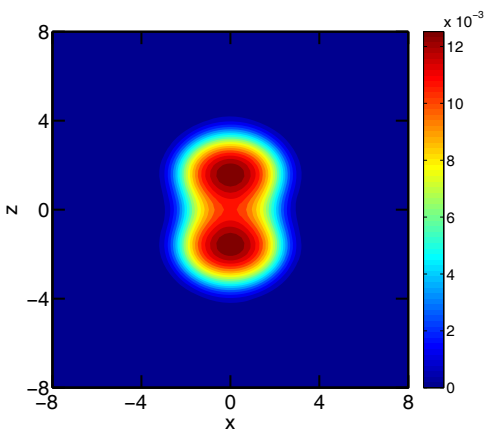

(b2) $|\phi(x, 0, z)|^{2}$

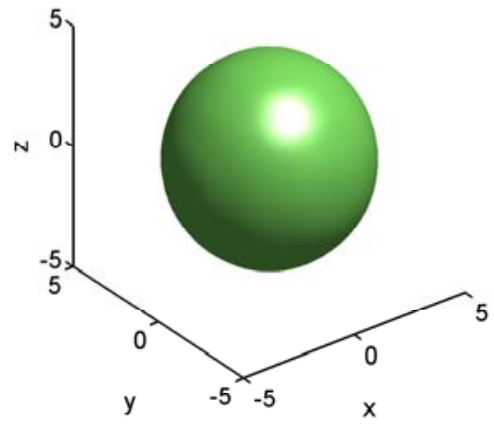

(c1) $|\phi(\mathbf{x})|=0.01$

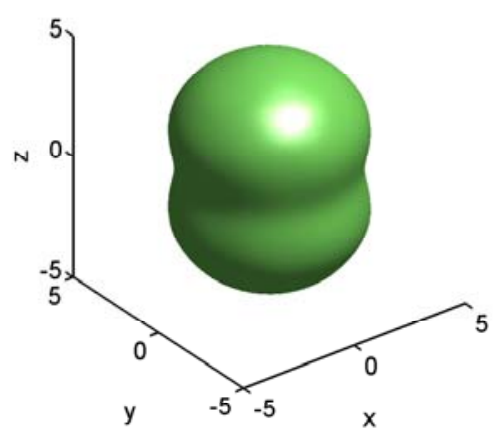

(c2) $|\phi(\mathbf{x})|=0.01$

Figure 2: The solution curves, the contour plots of $|\phi(x, 0, z)|^{2}$, and the isosurface plots of $|\phi(\mathbf{x})|=0.01$ for the ground state of (1.11) with $\mathbf{n}=(0,0,1)^{T}, \beta=401.432$, and $\lambda=64.22912$ for the harmonic potential $V(\mathbf{x})=\left(x^{2}+y^{2}+z^{2}\right) / 2$ (first row) and double-well potential $V(\mathbf{x})=\left(x^{2}+y^{2}+z^{2}\right) / 2+4 e^{-z^{2} / 2}$ (second row). 


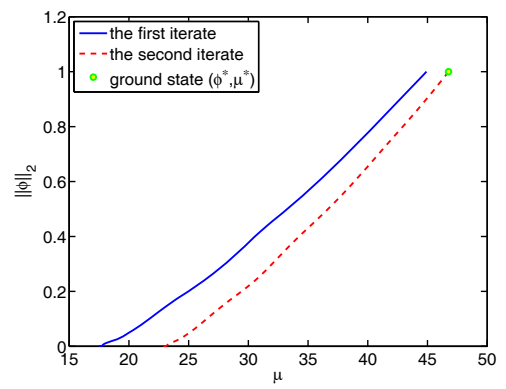

(a) solution curves

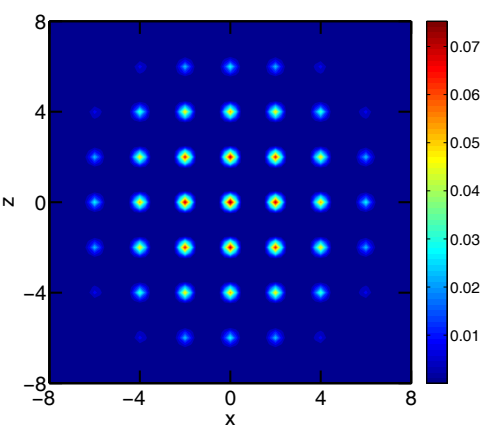

(b) $|\phi(x, 0, z)|^{2}$

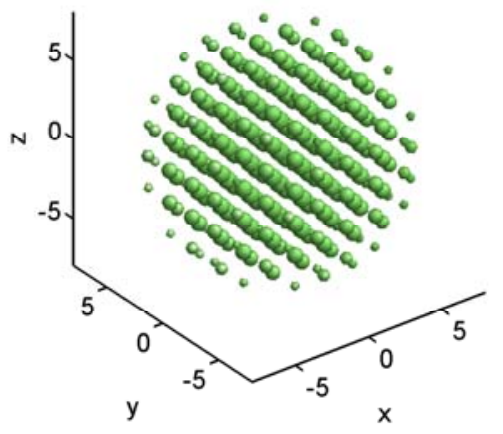

(c) $|\phi(\mathbf{x})|=0.04$

Figure 3: The solution curves, the contour plot of $|\phi(x, 0, z)|^{2}$, and the isosurface plot of $|\phi(\mathbf{x})|=0.04$ for the ground state of $(1.11)$, where $V(x)=\left(x^{2}+y^{2}+z^{2}\right) / 2+100\left(\sin ^{2}(\pi x / 2)+\right.$ $\left.\sin ^{2}(\pi y / 2)+\sin ^{2}(\pi z / 2)\right), \mathbf{n}=(0,0,1)^{T}, \beta=401.432$, and $\lambda=64.22912$. 


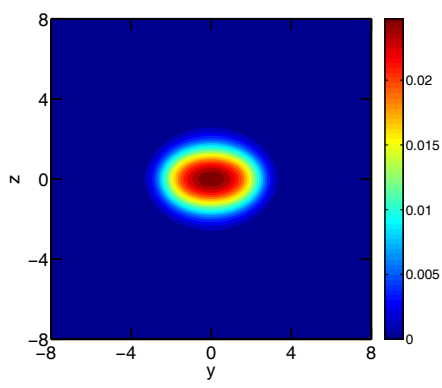

(1)

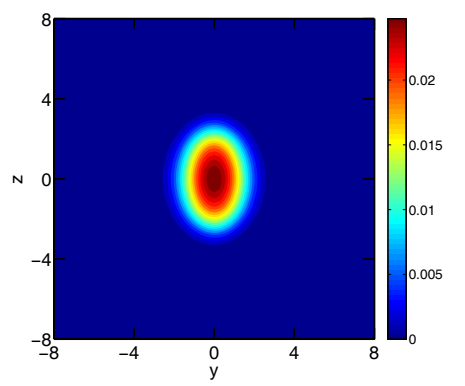

(1)

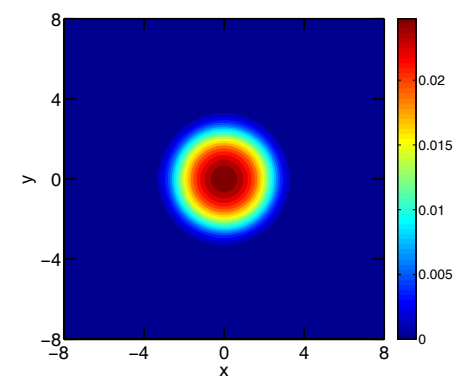

(2)

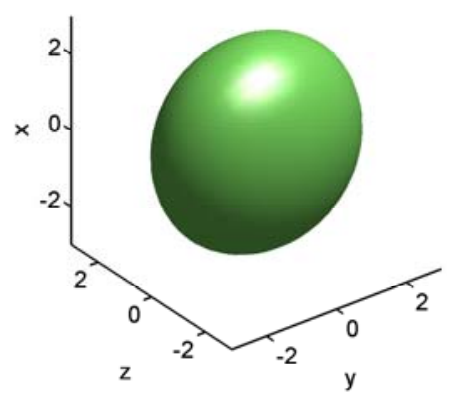

(3)

(a) $\mathbf{n}=(0,0,1)^{T}$

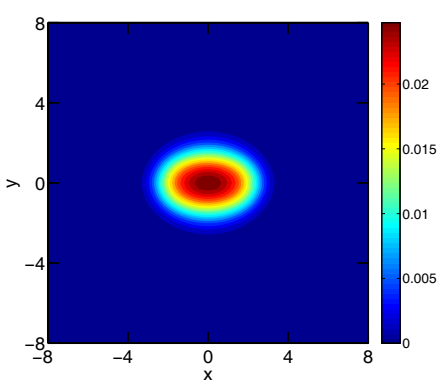

(2)

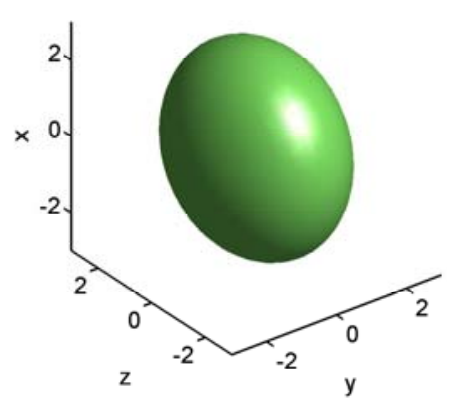

(3)

(b) $\mathbf{n}=(0,1,0)^{T}$

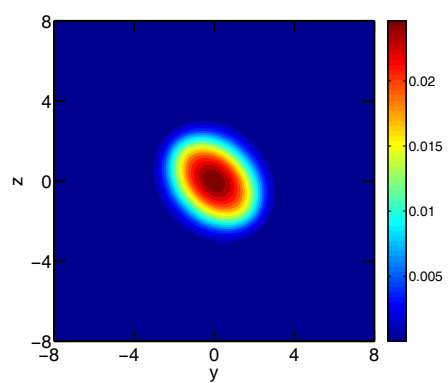

(1)

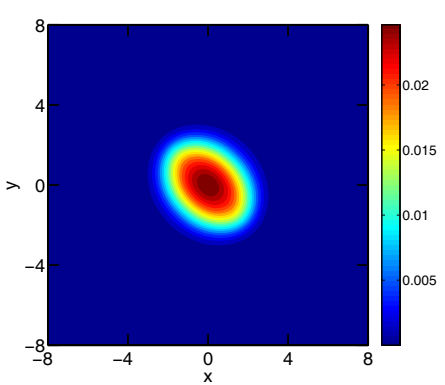

(2)

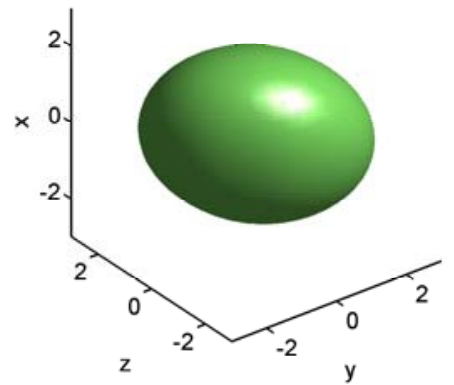

(3)

(c) $\mathbf{n}=(1,1,1)^{T} / \sqrt{3}$

Figure 4: The contour plots of $|\phi(0, y, z)|^{2},|\phi(x, y, 0)|^{2}$, and the isosurface plots of $|\phi(\mathbf{x})|=$ 0.08 for the ground state of (1.11) with $V(x)=\left(x^{2}+y^{2}+z^{2}\right) / 2, \beta=207.16, \lambda=-103.58$, and different dipolar directions $\mathbf{n}$. 


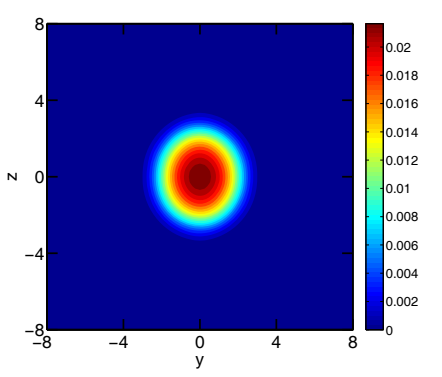

(1)

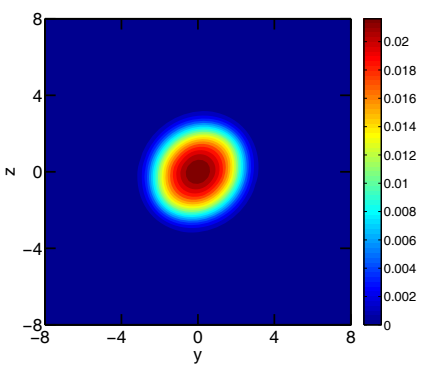

(1)

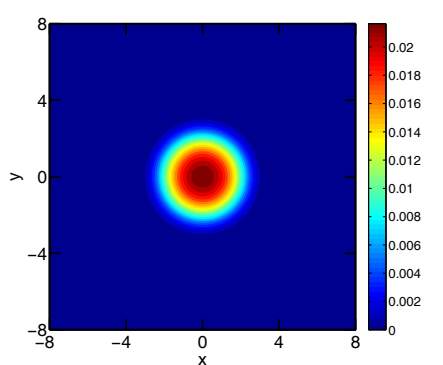

(2)

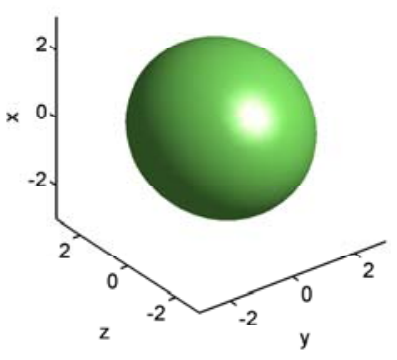

(3)
(a) $\mathbf{n}=(0,0,1)^{T}$

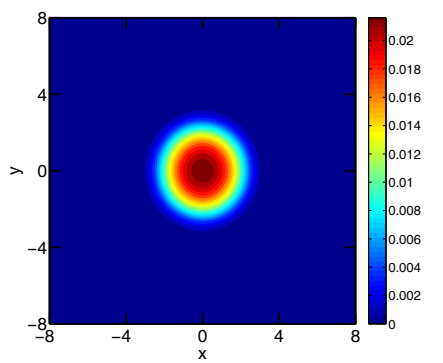

(2)

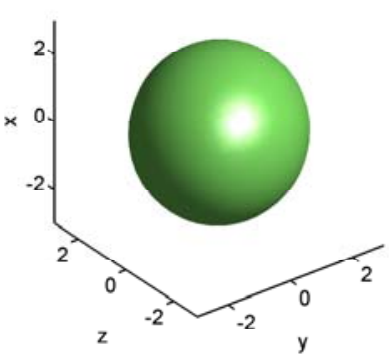

(3)

(b) $\mathbf{n}=(0,1,1)^{T} / \sqrt{2}$

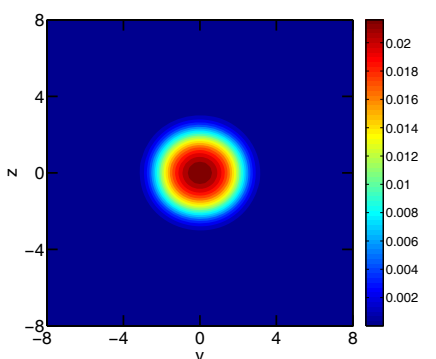

(1)

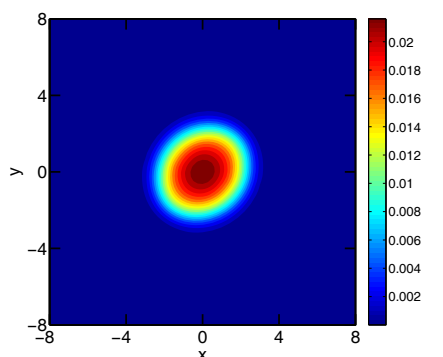

(2)

(c) $\mathbf{n}=(1,1,0)^{T} / \sqrt{2}$

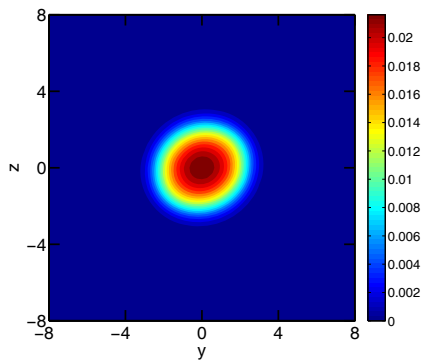

(1)

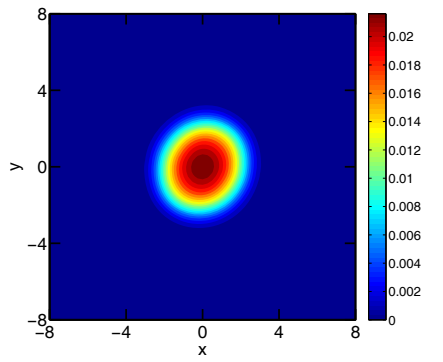

(2)

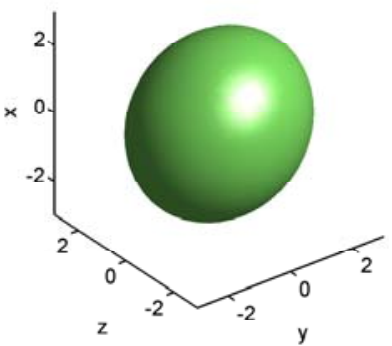

(3)

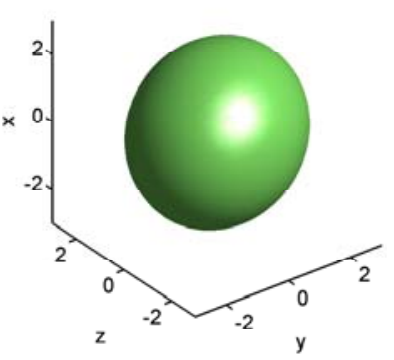

(3)

(d) $\mathbf{n}=(1,2,1)^{T} / \sqrt{6}$

Figure 5: The contour plots of $|\phi(0, y, z)|^{2},|\phi(x, y, 0)|^{2}$, and the isosurface plots of $|\phi(\mathbf{x})|=$ 0.08 for the ground state of (1.11) with $V(x)=\left(x^{2}+y^{2}+z^{2}\right) / 2, \beta=207.16, \lambda=51.79$, and different dipolar directions $\mathbf{n}$. 


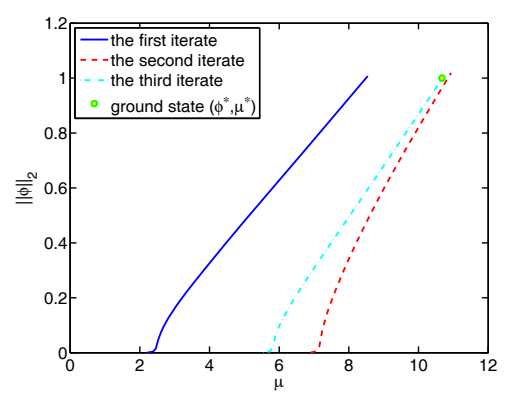

(a) solution curves, $\varepsilon_{2}=0.7, t=38916.27$

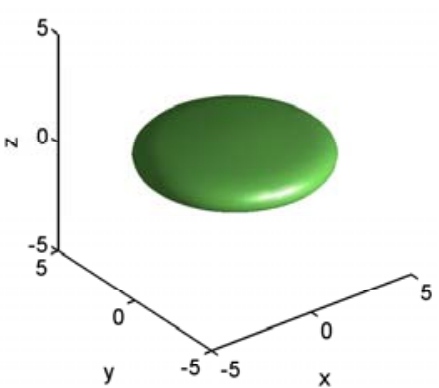

(b) $|\phi(\mathbf{x})|=0.05$

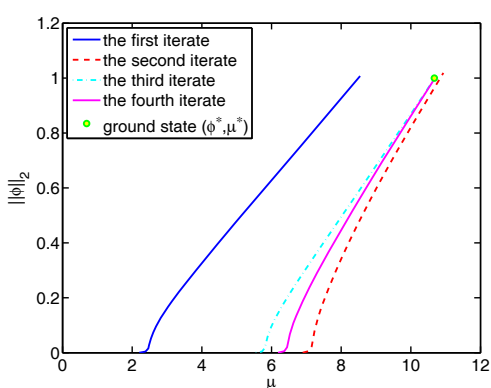

(c) solution curves, $\varepsilon_{2}=0.4, t=46317.83$

Figure 6: The solution curves and the isosurface plot of $|\phi(\mathbf{x})|=0.05$ for the ground state of $(1.11)$ with $V(\mathbf{x})=\left(x^{2}+y^{2}+25 z^{2}\right) / 2, \mathbf{n}=(0,0,1)^{T}, \beta=207.16$, and $\lambda=51.79$, where $t$ represents the total execution time (in seconds).

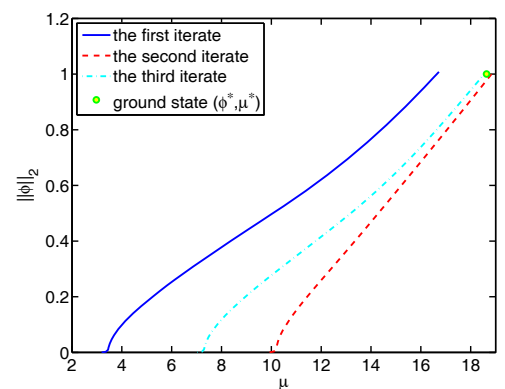

(a) solution curves, $\varepsilon_{2}=1.8, t=52090.05$

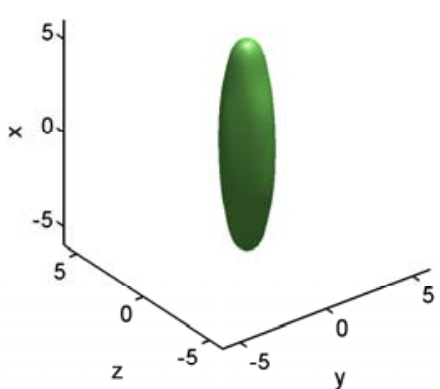

(b) $|\phi(\mathbf{x})|=0.05$

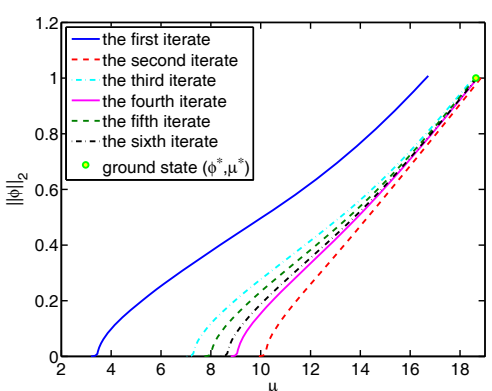

(c) solution curves, $\varepsilon_{2}=0.4, t=98733.63$

Figure 7: The solution curves and the isosurface plot of $|\phi(\mathbf{x})|=0.05$ for the ground state of (1.11) with $V(\mathbf{x})=\left(x^{2}+25 y^{2}+25 z^{2}\right) / 2, \mathbf{n}=(0,0,1)^{T}, \beta=207.16$, and $\lambda=51.79$, where $t$ represents the total execution time (in seconds).

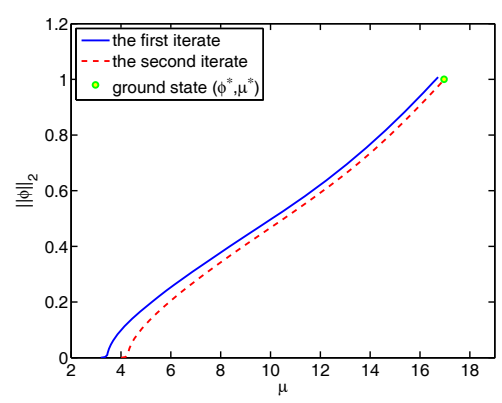

(a) solution curves

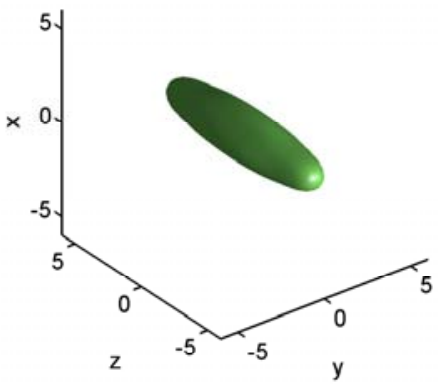

(b) $|\phi(\mathbf{x})|=0.05$

Figure 8: The solution curves and the isosurface plot of $|\phi(\mathbf{x})|=0.05$ for the ground state of (1.11) with $V(\mathbf{x})=\left(25 x^{2}+25 y^{2}+z^{2}\right) / 2, \mathbf{n}=(0,0,1)^{T}, \beta=207.16$, and $\lambda=51.79$. 


\section{References}

[1] A. Griesmaier, J. Werner, S. Hensler, J. Stuhler, T. Pfau, Bose-Einstein condensation of chromium, Phys. Rev. Lett. 94 (2005) 160401.

[2] M. S. Elioff, J. J. Valentini, D. W. Chandler, Subkelvin cooling NO molecules via "billiard-like" collisions with argon, Science 302 (2003) 1940-1943.

[3] D. Wang, J. Qi, M. F. Stone, O. Nikolayeva, H. Wang, B. Hattaway, S. D. Gensemer, P. L. Gould, E. E. Eyler, W. C. Stwalley, Photoassociative production and trapping of ultracold KRb molecules, Phys. Rev. Lett. 93 (2004) 243005.

[4] J. M. Sage, S. Sainis, T. Bergeman, D. DeMille, Optical production of ultracold polar molecules, Phys. Rev. Lett. 94 (2005) 203001.

[5] L. Santos, G. V. Shlyapnikov, P. Zoller, M. Lewenstein, Bose-Einstein condensation in trapped dipolar gases, Phys. Rev. Lett. 85 (2000) 1791-1794.

[6] K. Góral, K. Rzążewski, T. Pfau, Bose-Einstein condensation with magnetic dipoledipole forces, Phys. Rev. A 61 (2000) 051601(R).

[7] K. Góral, L. Santos, Ground state and elementary excitations of single and binary Bose-Einstein condensates of trapped dipolar gases, Phys. Rev. A 66 (2002) 023613.

[8] T. Lahaye, J. Metz, B. Fröhlich, T. Koch, M. Meister, A. Griesmaier, T. Pfau, H. Saito, Y. Kawaguchi, M. Ueda, $d$-Wave collapse and explosion of a dipolar Bose-Einstein condensate, Phys. Rev. Lett. 101 (2008) 080401.

[9] N. G. Parker, C. Ticknor, A. M. Martin, D. H. J. O'Dell, Structure formation during the collapse of a dipolar atomic Bose-Einstein condensate, Phys. Rev. A 79 (2009) 013617.

[10] P. Pedri, L. Santos, Two-dimensional bright solitons in dipolar Bose-Einstein condensates, Phys. Rev. Lett. 95 (2005) 200404.

[11] S. Yi, L. You, Expansion of a dipolar condensate, Phys. Rev. A 67 (2003) 045601.

[12] S. Yi, L. You, Trapped atomic condensates with anisotropic interactions, Phys. Rev. A 61 (2000) 041604(R).

[13] W. Bao, Y. Cai, H. Wang, Efficient numerical methods for computing ground states and dynamics of dipolar Bose-Einstein condensates, J. Comput. Phys. 229 (2010) 7874-7892. 
[14] R. Illner, O. Kavian, H. Lange, Stationary solutions of quasi-linear Schrödinger-Poisson systems, J. Differential Equations 145 (1998) 1-16.

[15] S. Costiner, S. Ta'asan, Simultaneous multigrid techniques for nonlinear eigenvalue problems: Solutions of the nonlinear Schrödinger-Poisson eigenvalue problem in two and three dimensions, Phys. Rev. E 52 (1995) 1181-1192.

[16] C.-S. Chien, B.-W. Jeng, Z.-C. Li, A time-independent approach for computing wave functions of the Schrödinger-Poisson system, Numer. Linear Algebra Appl. 15 (2008) $55-82$.

[17] Y. Zhang, Numerical study of vortex interactions in Bose-Einstein condensation, Commun. Comput. Phys. 8 (2010) 327-350.

[18] Y.-S. Wang, B.-W. Jeng, C.-S. Chien, A two-parameter continuation method for rotating two-component Bose-Einstein condensates in optical lattices, Commun. Comput. Phys. 13 (2013) 442-460.

[19] W. Bao, M.-H. Chai, A uniformly convergent numerical method for singularly perturbed nonlinear eigenvalue problems, Commun. Comput. Phys. 4 (2008) 135-160.

[20] Y. Cai, M. Rosenkranz, Z. Lei, W. Bao, Mean-field regime of trapped dipolar BoseEinstein condensates in one and two dimensions, Phys. Rev. A 82 (2010) 043623.

[21] W. Bao, N. B. Abdallah, Y. Cai, Gross-Pitaevskii-Poisson equations for dipolar BoseEinstein condensate with anisotropic confinement, SIAM J. Math. Anal. 44 (2012) 17131741.

[22] S.-L. Chang, C.-S. Chien, B.-W. Jeng, Computing wave functions of nonlinear Schrödinger equations: A time-independent approach, J. Comput. Phys. 226 (2007) 104-130.

[23] S.-L. Chang, C.-S. Chien, B.-W. Jeng, Liapunov-Schmidt reduction and continuation for nonlinear Schrödinger equations, SIAM J. Sci. Comput. 29 (2007) 729-755.

[24] S.-L. Chang, C.-S. Chien, Computing multiple peak solutions for Bose-Einstein condensates in optical lattices, Comput. Phys. Comm. 180 (2009) 926-947.

[25] H.-S. Chen, C.-S. Chien, Multilevel spectral-Galerkin and continuation methods for nonlinear Schrödinger equations, Multiscale Model. Simul. 8 (2009) 370-392. 
[26] H.-S. Chen, S.-L. Chang, C.-S. Chien, Spectral collocation methods using sine functions for a rotating Bose-Einstein condensation in optical lattices, J. Comput. Phys. 231 (2012) 1553-1569.

[27] E. L. Allgower, C.-S. Chien, Continuation and local perturbation for multiple bifurcations, SIAM J. Sci. Stat. Comput. 7 (1986) 1265-1281.

[28] S.-L. Chang, C.-S. Chien, A multigrid-Lanczos algorithm for the numerical solutions of nonlinear eigenvalue problems, Int. J. Bifurcation \& Chaos 13 (2003) 1217-1228.

[29] C.-S. Chien, S.-L. Chang, Application of the Lanczos algorithm for solving the linear systems that occur in continuation problems, Numer. Linear Algebra Appl. 10 (2003) $335-355$.

[30] C.-S. Chien, M.-S. Chen, Multiple bifurcation in the von Kármán equations, SIAM J. Sci. Comput. 18 (1997) 1737-1766.

[31] C.-S. Chien, Z. Mei, C.-L. Shen, Numerical continuation at double bifurcation points of a reaction-diffusion problem, Int. J. Bifurcation \& Chaos 8 (1997) 117-139.

[32] T. Lahaye, C. Menotti, L. Santos, M. Lewenstein, T. Pfau, The physics of dipolar bosonic quantum gases, Rep. Prog. Phys. 72 (2009) 126401. 